\title{
Isotropy of an upper limb exoskeleton and the kinematics and dynamics of the human arm
}

\author{
Joel C. Perry ${ }^{\mathrm{a}}$, Janet M. Powell ${ }^{\mathrm{b}}$ and Jacob Rosen ${ }^{\mathrm{c} *}$ \\ ${ }^{a}$ Department of Mechanical Engineering; ${ }^{b}$ Department of Rehabilitation Medicine, University of Washington, Seattle Washington, USA; \\ ${ }^{c}$ Department of Computer Engineering, University of California at Santa Cruz, Santa Cruz, California, USA
}

(Received 12 December 2008; final version received 11 March 2009)

\begin{abstract}
The integration of human and robot into a single system offers remarkable opportunities for a new generation of assistive technology. Despite the recent prominence of upper limb exoskeletons in assistive applications, the human arm kinematics and dynamics are usually described in single or multiple arm movements that are not associated with any concrete activity of daily living (ADL). Moreover, the design of an exoskeleton, which is physically linked to the human body, must have a workspace that matches as close as possible with the workspace of the human body, while at the same time avoid singular configurations of the exoskeleton within the human workspace. The aims of the research reported in this manuscript are (1) to study the kinematics and the dynamics of the human arm during daily activities in a free and unconstrained environment, (2) to study the manipulability (isotropy) of a 7-degree-of-freedom (DOF)-powered exoskeleton arm given the kinematics and the dynamics of the human arm in ADLs. Kinematic data of the upper limb were acquired with a motion capture system while performing 24 daily activities from six subjects. Utilising a 7-DOF model of the human arm, the equations of motion were used to calculate joint torques from measured kinematics. In addition, the exoskeleton isotropy was calculated and mapped with respect to the spacial distribution of the human arm configurations during the 24 daily activities. The results indicate that the kinematic joint distributions representing all 24 actions appear normally distributed except for elbow flexion-extension with the emergence of three modal centres. Velocity and acceleration components of joint torque distributions were normally distributed about $0 \mathrm{Nm}$, whereas gravitational component distributions varied with joint. Additionally, velocity effects were found to contribute only $1 / 100$ th of the total joint torque, whereas acceleration components contribute $1 / 10$ th of the total torque at the shoulder and elbow, and nearly half of the total torque at the wrist. These results suggest that the majority of human arm joint torques are devoted to supporting the human arm position in space while compensating gravitational loads whereas a minor portion of the joint torques is dedicated to arm motion itself. A unique axial orientation at the base of the exoskeleton allowed the singular configuration of the shoulder joint to be moved towards the boundary of the human arm workspace while supporting $95 \%$ of the arm's workspace. At the same time, this orientation allowed the best exoskeleton manipulability at the most commonly used human arm configuration during ADLs. One of the potential implications of these results might be the need to compensate gravitational load during robotic-assistive rehabilitation treatment. Moreover, results of a manipulability analysis of the exoskeleton system indicate that the singular configuration of the exoskeleton system may be moved out of the human arm physiological workspace while maximising the overlap between the human arm and the exoskeleton workspaces. The collected database along with kinematic and dynamic analyses may provide a fundamental basis towards the development of assistive technologies for the human arm.
\end{abstract}

Keywords: assistive robotics; daily activities; exoskeleton; human arm; upper limb; kinematics; dynamics; isotropy; manipulability; equations of motion; workspace; rehabilitation robotics

\section{Introduction}

Nature provides a wide spectrum of solutions for a skeleton, the system that provides physical support for the organism and facilitates locomotion. Skeletal systems may be divided into three types: (1) Internal - endoskeletons, (2) External - exoskeletons and (3) Fluid based - hydrostatic skeletons. The endoskeleton is an internal skeletal system consisting of rigid structures (bones - mineralised or ossified, e.g. in humans) or semi-rigid structures (cartilage) that are incorporated into joints or substantiate bones completely (e.g. in sharks). The elements of the endoskeleton system are connected with each other by ligaments and through tendons to the muscular system. The exoskeleton, in contrast, is an external skeletal system utilised to both support and protect the body (e.g. arthropods, such as spiders, insects, lobster, crab, shrimp). A hydrostatic skeleton, or hydroskeleton, is a structure found in soft-bodied animals which consists of a fluid-filled cavity - the coelom, surrounded by muscles. The surrounding muscles encapsulating the pressurised coelom are used to change an organism's shape and produce movements, such as burrowing (earthworm) and swimming (squid and jellyfish).

In medicine, any device implanted or attached to the human body may be classified as either a prosthesis or an

*Corresponding author. Email: rosen@ucsc.edu 
orthosis. A limb prosthesis (plural: prostheses) is a device that substitutes a missing part of a limb whereas a limb orthosis (plural: orthoses) is a device that attaches to a limb, or the torso, to support the function or correct the shape of an existing limb. If actuated, each of these devices may be considered a type of wearable robot. The exoskeleton robot, serving as an assistive device, is worn by the human (orthosis) and functions as a human-amplifier (see Figure 2(a)). Its joints and links correspond to those of the human body, and its actuators share a portion of the external load with the operator. Developing a powered exoskeleton that would serve a human operator adequately during daily activities requires a profound understanding of the kinematics and dynamics of the human arm during these activities, and is beyond the anthropometric information that has been widely known for several decades.

The US Census Bureau reported that 10.1 million individuals require assistance with one or more activities of daily living (ADLs) (US Census Bureau 2001). Statistics of persons suffering the effect of first or recurrent strokes are equally daunting at more than 5 million (Thom et al. 2006). For most people with neuromuscular disorders (NMDs) and central nervous system (CNS) injuries, muscle weakness is the primary cause of disability. Wearable robotics or exoskeleton may address some of the debilitating effects of muscle weakness within the myopathic and neuropathic population as well as in temporary utilization of such systems for rehabilitation. Although humans possess naturally developed algorithms for control of movement, they are limited in muscle strength. In contrast, robotic manipulators can perform tasks that require large forces; however, their artificial control algorithms do not provide the flexibility to perform in a wide range of conditions while preserving the same quality of performance as humans. Along these lines, it is believed that the integration of the human and robot into a single collaborative system will lead to a solution that will benefit from the advantages offered by each subsystem.

A wide variety of exoskeleton systems both for upper limbs (Bergamasco Frisoli et al. 2007; Carignan et al. 2008; Kiguchi et al. 2006; Klein et al. 2008; Mihelj et al. 2007; Stienen et al. 2007; Tsagarakis and Caldwell 2003; Romilly 1994) and lower limbs (Banala et al. 2007; Blaya and Herr 2004; Ferris et al. 2007; Kawamoto et al. 2003; Zoss et al. 2005) with various human-machine interfaces have been developed (for review see Casolo et al. 2008; Guizzo and Goldstein 2005; IJHR 2007; Pons 2007; Van der Loos and Reinkensmeyer 2008). The design of an exoskeleton as a wearable device should rely not only on anthropometric information of the human body (Department of Defense 1991; Department of Transportation 1975) but also on comprehensive information regarding human body kinematics and dynamics. A prior one-subject pilot study on kinematics and dynamics of human arm motion was previously conducted by the authors and reported by Rosen et al. 2005. In this first study, many of the reach and grasp tasks were simulated without the use of props, such as drawers, cupboards, combs, toothbrushes and food. In the present study, measures were taken to ensure that provided props were appropriate for the action so that motion trajectories would be as natural as possible. Another significant change was in the 7-degree-of-freedom (DOF) model representation of forearm rotation. Due to the simplification involved in representing the human arm as a 7-DOF model, inaccuracies are inevitable. In the first pilot study, forearm rotation was placed at the wrist and mathematically was only moving the mass of the hand, whereas in the present study this rotation has been reallocated to the elbow and the act of internal/external rotation moves mass of both the hand and the forearm. The first election yields an underestimation of ADL joint torques, while the latter yields an overestimation and the actual value lies somewhere between the two bounds.

The aims of the research reported in this manuscript are (1) to fill a current gap in literature regarding the range and distribution of kinematics and dynamic of the human arm during daily activities and (2) to study the manipulability (isotropy) of a 7-DOF-powered exoskeleton arm given the kinematics and the dynamics of the human arm. The study is part of an on-going research effort towards the development and the utilisation of a 7-DOF-powered exoskeleton for the human arm (Ettore et al. 2006; Rosen and Perry 2006; Jacob et al. 1999, 2001; Perry and Rosen 2006; Perry et al. 2007).

\section{Methods and tools}

\section{A. Experimental protocol}

The kinematics of the human arm were collected during 24 daily activities from six subjects in the age of 20 to 41 years. Mean and standard deviation (SD) height, weight and age for the subjects were $1.72+/-0.08$ metres, 76.2 $+/-23.1 \mathrm{~kg}$, and $26.2+/-7.7$ years, respectively. Of the six subjects, three were males and three females. Arm kinematic data were collected using a VICON motion capture system (Vicon Inc.) at a sampling frequency of $120 \mathrm{~Hz}$. Raw optical data were captured synchronously from 12 cameras and filtered with a Woltring quintic spline filter having a mean square error of $20 \mathrm{~mm}^{2}$.

Reflective markers of $14 \mathrm{~mm}$ diameter were attached to the right (dominant) arm of each subject at seven key anatomical locations (Figure 1a). Individual models were calibrated for each subject and marker set for use during VICON post-processing of subject data. Subjects were instructed to perform three to six repetitions of each arm activity. The 24 arm activities were divided into four subgroups: (i) seven reaching tasks, (ii) eight functional ADL tasks, (iii) four eating and drinking tasks and (iv) five hygiene (i.e. grooming) tasks. Specific human arm activities 

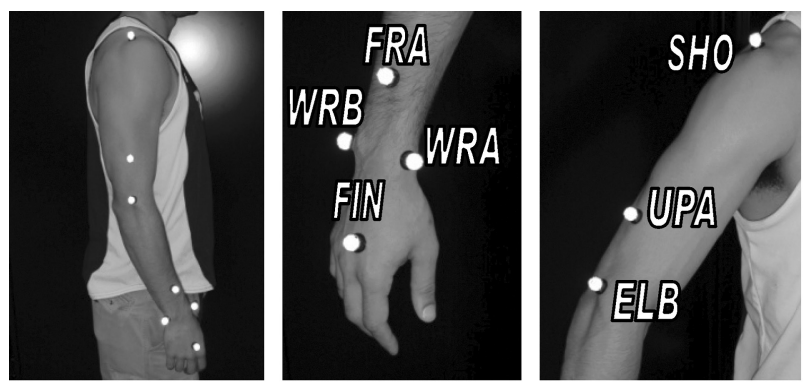

(a)

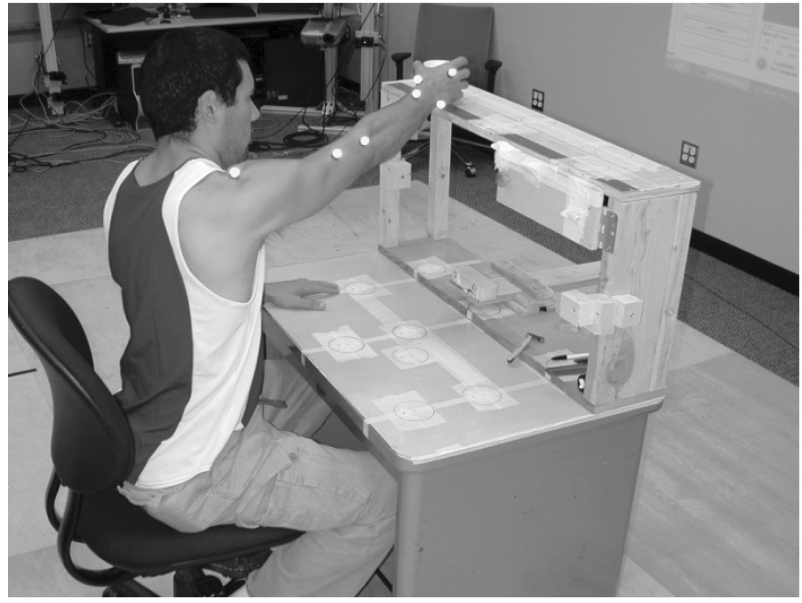

(b)

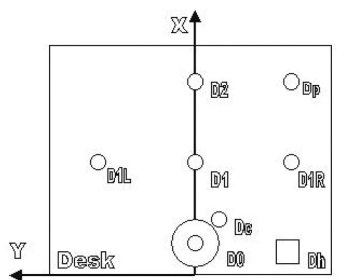

(c)

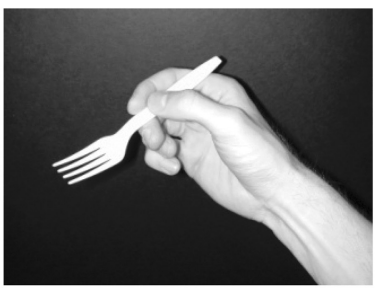

(d)
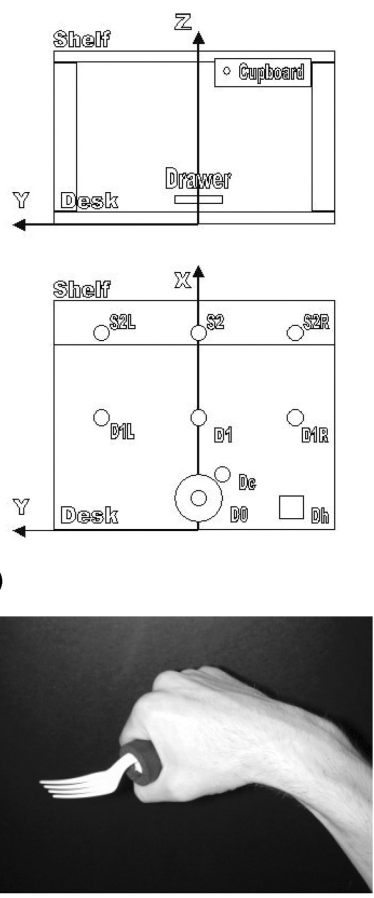

(e)

Figure 1. Overview of the experimental setup in the motion capturing lab. (a) Retro-reflective markers were placed on the subjects at the following seven anatomical locations: acromioclavicular joint (SHO), lateral upper arm (UPA), lateral elbow epicondyle (ELB), lateral forearm (FRA), radial (WRA) and ulnar (WRB) styloid processes at the wrist, and between 2nd and 3rd metacarpals. (b) Overview of the desk and shelf (C), Desk (D) and shelf (S) locations as defined in the ADL study were divided into three medial-lateral, three anteriorposterior, and two superior-inferior positions. A top view of the desktop (left) shows eight target desk locations, more heavily concentrated in the right-hand plane. The three shelf locations were located at the same x-coordinate as desk locations D2 and Dp, only 0.44 metres above the desktop in the positive z-direction (bottom-right). Also attachable to the desk and shelf were a wood drawer, a cupboard and a door (top-right). During action 16, normal eating with a fork, subjects were instructed to eat using a standard three-fingered grasp (d), while in action 17 , subjects used a fork that had been fit with a standard grip buildup to aid in myopathic (power) grasp (e).

in each subgroup were selected based on previous surveys of the disabled community indicating the desired tasks and functionality of powered orthotic and robotic rehabilitation devices (Ramanathan 1994; Stanger et al. 1994). Seven of the 24 actions were repeated each for six trials to look at intra- and inter-subject variations during reaching, functional, eating and hygiene tasks. All subgroup tasks with the exception of action 8 (opening and closing a door) were performed from a seated position; action 8 was performed in a standing posture with the midline of the body comfortably aligned with the doorknob. During seated tasks, the subject sat down at a desk and manipulated objects in the workspace between predefined desk (D) and shelf (S) locations (see Figure 1c).

Desktop locations were anteriorly divided into three regions (D0, D1 and D2) as shown in Figure 1(c), with lateral components at the D1 level (D1L, D1R). Additional desk- top locations were specified for objects used during specific tasks such as drinking from a cup (Dc), picking up a phone or pitcher (Dp) or the resting location of the hand (Dh). Left, central and right positions were located 0.25 metres to the left of the midline of the body, at the midline of the body and 0.30 metres to the right of the midline, respectively. The leftmost target position was chosen so that it placed the object directly in front of the lateral edge of the left shoulder for a 99th percentile individual. D0, D1 and D2 locations were placed 0.10 metres, 0.25 metres and 0.46 metres, respectively each from the front edge of the desk. Shelf locations are labeled S2L, S2 and S2R, for the left, central and right positions (Figure 1c, bottom-right). All objects were placed with respect to each desk or shelf location so that the centre of the handgrip during object grasping and releasing was positioned vertically over each specified location. Desk and shelf heights were 0.76 and 
1.20 metres, respectively from the floor. Additionally, all individuals sat in the same chair with a fixed seat-to-floor height of 0.50 metres to properly represent the effect of individual subject height on joint kinematics and dynamics while operating in a typical fixed environment.

Opening and closing a door was simulated using an actual doorknob fixed 0.77 metres from the hinge, at a height of 0.95 metres with respect to the floor and was allowed $45^{\circ}$ of travel to the 'open' state. The cupboard doorknob was positioned 0.38 metres above the desk, 0.38 metres from the front of the desk, 0.14 metres to the right of the midline and was allowed about $95^{\circ}$ of travel to the 'open' state. The drawer was placed along the midline at a distance 0.38 metres from the front of the desk. Drawer motion underwent 0.35 metres of travel in the negative $\mathrm{x}$-direction to the 'open' state. The drawer handle was $76 \mathrm{~mm}$ above the desktop, having a cylindrical gripping surface of 6.3 $\mathrm{mm}$ diameter by $57.1 \mathrm{~mm}$ length. Without instruction, all subjects naturally gripped the handle using an over-handed grasp.

In addition to the desk and shelf locations outlined in Figure 1, a rest pose (RP) for the arm was defined as the arm resting comfortably along the subject's side. The selected tasks from each subgroup and the variations for each task are illustrated as follows:

1. Reaching (actions 1-7): Placing hand on desk (RP to $\mathrm{Dh}$ ), moving an object between desktop positions (D1R to D1L, D0 to D2), moving an object between shelf positions (S2L to S2R), combination shelf/desktop object motions (D1R to S2L, D1L to S2R, D1 to S2).

2. Functional ADLs (actions 8-15): Opening and closing a door/cabinet/drawer, answering a table-mounted phone (Dp), overhand/underhand stirring with a utensil (D1), pouring from a pitcher to a cup (Dp to Dc) and pouring from a cup to a pitcher (D1R to D1).

3. Eating and drinking (actions 16-19): Normal eating with a fork (D0), powered grasp eating with a fork (D0), eating with right hand (D0) and drinking from a cup (Dc).

4. Hygiene (actions 20-24): Combing the hair, washing the face, shaving the face, brushing the teeth and washing the neck.

During the bulk of the above-mentioned activities, subjects interacted with various small and free objects as directed by the nature of each activity, and in a few cases interacted with partially constrained objects. It is assumed that all motions were made in accordance with the existing object constraints (e.g. door and cupboard hinges) such that no external forces or torques, other than those due to inertial and gravitational effects, were applied on the human by the object. During contact with constrained objects, although it is possible for large external forces to be applied on the arm if the constraint-induced trajectory is not followed, the data acquisition system and the mathematical dynamic model have no knowledge of this phenomenon. The dynamic model computes net joint torques based on measured angular position, velocity and acceleration about 7 DOFs. To minimise the potential for unmodeled dynamics, subjects were allowed to explore fully all constrained objects to familiarise themselves with the resulting constraint-induced motions.

With the exception of actions 1 and 8, every action began from an initial arm position in which the hand was resting at $\mathrm{Dh}$ and the upper and lower arms were relaxed. Action 1 began from a seated position with the arm in the resting pose, along the side of the subject and chair. For reaching tasks and functional opening/closing tasks, the hand begins from rest at $\mathrm{Dh}$, moves the object from the object starting position (position 1) to an intermediate position (position 2). Leaving the object at position 2, the hand returns to rest at $\mathrm{Dh}$ for one second and then repeats the action in reverse moving the object from position 2 back to position 1 . In the case of opening and closing actions, positions 1 and 2 refer to the closed and open states, respectively, of the objects. The objects used during each action and the positions associated with each action are shown in Table 1. The one-second time interval between motions

Table 1. Objects, target locations and segmentation information for each action. An object was moved from position 1 to position 2 and back to position 1 in each action (No.). Data were segmented in either 1 or 2 segments triggered by motion of the trigger listed (segment trigger). The normal (norm) or myopathic (myop) grasp is the only distinguishing factor between actions 16 and 17.

\begin{tabular}{rrllcl}
\hline & $\begin{array}{c}\text { ADL } \\
\text { type }\end{array}$ & Object & $\begin{array}{l}\text { Position 1/ } \\
\text { Position 2 }\end{array}$ & $\begin{array}{c}\text { No. of } \\
\text { Segments. }\end{array}$ & $\begin{array}{l}\text { Segment } \\
\text { trigger }\end{array}$ \\
\hline 1 & R & Hand & RP/Dh & 2 & Hand \\
2 & R & Cup & D1R/D1L & 2 & Cup \\
3 & R & Cup & D0/D2 & 2 & Cup \\
4 & R & Cup & S2L/S2R & 2 & Cup \\
5 & R & Cup & D1R/S2L & 2 & Cup \\
6 & R & Cup & D1L/S2R & 2 & Cup \\
7 & R & Cup & D1/S2 & 2 & Cup \\
8 & F & Door & Closed/Open & 2 & Door \\
9 & F & Cupboard & Closed/Open & 2 & Cupboard \\
10 & F & Drawer & Closed/Open & 2 & Drawer \\
11 & F & Table Phone & Dp/R. Ear & 1 & Phone \\
12 & F & Short Utensil & Dh/D1 & 1 & Hand \\
13 & F & Long Utensil & Dh/D1 & 1 & Hand \\
14 & F & Pitcher & Dp/Dc & 1 & Hand \\
15 & F & Cup & D1R/D1 & 1 & Hand \\
16 & E & Fork (norm) & Dh/D0 & 1 & Hand \\
17 & E & Fork (myop) & Dh/D0 & 1 & Hand \\
18 & E & Hand & Dh/D0 & 1 & Hand \\
19 & D & Cup & Dc/Mouth & 1 & Cup \\
20 & H & Comb & Dh/Hair & 1 & Hand \\
21 & H & Hand & Dh/Face & 1 & Hand \\
22 & H & Man. Razor & Dh/Face & 1 & Hand \\
23 & H & Toothbrush & Dh/Mouth & 1 & Hand \\
24 & H & Hand & Dh/Neck & 1 & Hand \\
\hline
\end{tabular}


was incorporated to facilitate the automated data segmentation process. Information about segments listed in the table and the segmentation process are further explained in Section II.B.

The arm actions associated with eating with a fork had two variations. Action 16 involved normal fork handling where a three-fingered grasp was used to orient the fork (Figure 1(d)). In action 17, a powered grasp of the fork (Figure 1(e)) was used to emulate a type of grasp often necessary for patients who suffer from one of several neurological disorders. The handle of the fork in this case was fitted with a typical buildup used to facilitate grasping in the patient population having such disorders.

For each arm motion, Euler joint angles for the 7 DOFs of the human arm were calculated based on the Cartesian coordinates of each marker. This transformation was performed by the VICON system by matching marker trajectories to a 7-DOF model of the arm. The arm model incorporates anthropometric data, such as segment lengths and epicondylar widths, which were measured directly from the subject under study, and then allows the calibration to optimise model parameters based on a calibration trial. Such measures and calibrations were performed for each of the six subjects to obain the best fit between measured data and the VICON marker model.

The 7-DOF model is composed of a 3-DOF shoulder, a 2-DOF elbow and a 2-DOF wrist. The order of Euler angle rotations in the model is YXZ as follows: shoulder flexion-extension (SF), shoulder abductionadduction (SA), shoulder internal-external rotation (SR), elbow flexion-extension (EF), forearm rotation (FR), wrist flexion-extension (WF) and wrist radial-ulnar deviation (WD). The rotation about the long axis of the forearm was considered a rotation of the forearm at the elbow, rather than a rotation of the hand at the wrist. This election is further addressed in the discussion. The values defined as positive in the study are motions, or torques that cause motion, in the following directions: shoulder adduction, shoulder extension, shoulder internal rotation, elbow extension, forearm pronation, wrist flexion and wrist ulnar deviation.

The motion capture software (Vicon iQ 2.5, VICON) offers a real-time reconstruction fit of the markers with the calibrated subject arm model. The real-time model reconstruction was then directly compared with the subject under study during each trial capture with the VICON system to ensure the appropriate transformation between marker coordinates and the calculated joint angles.

\section{B. Kinematics}

\section{Human arm kinematics}

The human arm kinematics data were an output in local Euler coordinates directly from the VICON software. Position data were filtered using a 4th-order Butterworth filter with a cutoff frequency of 5 hertz. Joint velocities and accelerations were each computed as two-point differences from raw position and computed velocity data, respectively, and then filtered with the same 4th-order Butterworth filter.

Action durations for each trail were normalised over the trial length, and depending on the specifics of the action were composed of either one or two data segments (No. of Segment), as indicated in Table 1. Actions involving movement of an object from position 1 to position 2 and then back were deemed to have two separate segments, each beginning and ending with the onset and disappearance of motion of an object (i.e. the cup, cupboard or hand). The data were then resampled using a polyphase FIR filter and the two segments were concatenated together. Resampling took place prior to concatenation to eliminate side lobe effect of the (resampling) process and was performed such that each trial contained 1000 data points. Mean and SD curves for each action could then be computed both for individual subjects and across all six subjects. Moreover, the distribution of elbow and wrist locations were computed and plotted in polar coordinates along with the isotropy of the exoskeleton arm.

\section{Exoskeleton kinematics - Isotropy and singular con- figurations}

The analysis of the exoskeleton arm was based on a formal kinematic analysis of a serial mechanism while using the DH parameters (See Table 2). The transformation from frame 0 in which the $X_{0}$ axis is pointing to the side, $Y_{0}$ axis is pointing to the front and $Z_{0}$ axis is pointing up to frame 1 base (the first frame of the exoskeleton) required

Table 2. The DH parameter of the exoskeleton right arm where $i$ is the axis (joint) number which is aligned with $\hat{Z}_{i}$ coordinate system located at each joint, $a_{i}$ is the distance from $\hat{Z}_{i}$ to $\hat{Z}_{i+1}$ measured along $\hat{X}_{i}, \alpha_{i}$ is the angle between $\hat{Z}_{i}$ and $\hat{Z}_{i+1}$ measured about $\hat{X}_{i}, d_{i}$ is the distance from $\hat{X}_{i-1}$ to $\hat{X}_{i}$ measured along $\hat{Z}_{i}$, $\theta_{i}$ is the angle between $\hat{X}_{i-1}$ and $\hat{X}_{i}$ measured about $\hat{Z}_{i}$ and $\varphi_{i}$ are the offset angles to $\theta_{i}$ that will position the arm perpendicular to the ground and parallel to the human body in a standing position. Figure 2(b) defines the rotation along the $\hat{Z}_{i}$ axis for each one of the seven joints. Once the $\hat{Z}_{i}$ is defined with respect to the mechanism, the parameters are derived according to their classical definitions listed above.

\begin{tabular}{cccccccc}
\hline$i-1$ & $i$ & $i+1$ & $a_{i-1}$ & $\alpha_{i-1}$ & $d_{i}$ & $\theta_{i}$ & $\varphi_{i}$ \\
\hline & 1 & 2 & 0 & $90^{\circ}$ & 0 & $\theta_{1}$ & $-32.94^{\circ}$ \\
1 & 2 & 3 & 0 & $90^{\circ}$ & 0 & $\theta_{2}$ & $-28.53^{\circ}$ \\
2 & 3 & 4 & 0 & $90^{\circ}$ & $L_{\text {Upper limb }}$ & $\theta_{3}$ & $53.60^{\circ}$ \\
3 & 4 & 5 & 0 & $90^{\circ}$ & 0 & $\theta_{4}$ & $-90^{\circ}$ \\
4 & 5 & 6 & 0 & $90^{\circ}$ & $L_{\text {Lower limb }}$ & $\theta_{5}$ & $0^{\circ}$ \\
5 & 6 & 7 & 0 & $90^{\circ}$ & 0 & $\theta_{6}$ & $0^{\circ}$ \\
6 & 7 & & 0 & $90^{\circ}$ & 0 & $\theta_{7}$ & $0^{\circ}$ \\
\hline
\end{tabular}


the following three consecutive Euler rotation with respect to the moving reference frame.

$$
{ }_{1}^{0} T=\operatorname{Rot}\left(\mathrm{X}_{0}, 42.5^{\circ}+90^{\circ}\right) \operatorname{Rot}\left(\mathrm{Y}^{\prime}, 45^{\circ}\right) \operatorname{Rot}\left(\mathrm{Z}^{\prime \prime}, 90^{\circ}\right) .
$$

Positions of the elbow joint ${ }^{0} P_{\text {Elbow }}$ and the wrist joint ${ }^{0} P_{\text {Wrist }}$ in space with respect to frame 0 are defined by Equations (2) and (3) respectively

$$
\begin{aligned}
& { }^{0} P_{\text {Elbow }}={ }_{1}^{0} T_{2}^{1} T_{3}^{2} T\left[\begin{array}{llll}
0 & 0 & 0 & 1
\end{array}\right]^{T} \text { and } \\
& { }^{0} P_{\text {Wrist }}={ }_{1}^{0} T_{2}^{1} T_{3}^{2} T_{4}^{3} T_{5}^{4} T\left[\begin{array}{llll}
0 & 0 & 0 & 1
\end{array}\right]^{T},
\end{aligned}
$$

where ${ }_{i+1}^{i} T$ is the homogeneous transformation from coordinate system $i$ to coordinate system $i+1$ as defined by the DH parameters that are listed in Table 2.

The Jacobian matrices can be derived for the individual joints of shoulder, elbow and wrist. The isotropy of the joints was defined by Equation (4) as the ratio of the lowest to the highest Eigen value of the Jacobian matrix. Using Equation (4) to address the upper and lower arms separately, the joint isotropies were calculated from both base-to-elbow and elbow-to-wrist Jacobians. This approach was used to avoid the calculation of the entire 7DOF mechanism. As a result, the Jacobian matrix is always a squared matrix. The singular configuration of shoulder and wrist joints is therefore related to the classical 'gimble lock' (See Figures 2e, $2 \mathrm{f}$ and $2 \mathrm{~g}$ ). Note that due to anatomical constrains of the wrist joint a gimble lock is not achieved.

$$
\text { Isotropy }=\frac{\lambda_{\min }}{\lambda_{\max }} .
$$

The isotropy is a measure of directional uniformity of the manipulability ellipsoid. The isotropy values range between 0 (singular configuration) and 1 (completely isotropic). The advantage of using the isotropy as a performance index is the ability to plot iso-performance lines in space and map them with respect to the spatial statistical distribution of the human arm position (e.g. elbow and wrist positions). Singularity, as expressed from the mechanism perspective, is a device configuration where a DOF is lost or compromised as a result of the alignment of two rotational axes. In the development of a 3DOF spherical joint, such as the shoulder joint and the wrist joint, the existence or non-existence of singularities will depend entirely on the desired reachable workspace, where spherical workspaces equal to or larger than a hemisphere will always contain singular positions. The challenge is to place the singularity in an unreachable or near-unreachable location, such as the edge of the physiological workspace. This challenge can be met by orienting the base of the exoskeleton and placing the singular configuration of the shoulder joint (three intersecting rotational axes) at the edge of the shoulder joint workspace.
The elbow joint of the exoskeleton has an inherent singular configuration that takes place when the arm is fully stretched. As a result, a singular configuration due to the elbow joint angle could be obtained at any point within the human arm workspace. Theoretically, the three intersecting rotation axes of the exoskeleton wrist joint may also introduce singular configuration. However, due to the limited workspace of the human wrist, this singularity will never be introduced in the physiological range of motion.

In the field of robotics, the tendency is to avoid singular configurations of the robotic system either through the mechanism design or by designing trajectories that will avoid singular or even close to singular arm configurations. On the other hand, the human body utilises its singular configurations as an efficient way to transmit loads. For example, when pushing or pulling heavy loads the arms tend to stretch and lock the elbow joint in a singular configuration as a way to transfer the load through the skeleton with minimal assistance from the flexor and extensor muscle groups. Similarly, locking the knee joint in a standing posture transfers the body weight as well as additional external load to the ground with minimal efforts from the flexor and extensor muscles of the knee joint. However, from the design perspective of the exoskeleton as a system that is mechanically linked to the human body, the goal is to minimise singular configurations of the exoskeleton within the physiological workspace of the human arm. This is particularly true for the shoulder joint but not for elbow and wrist joints for reasons that have been already mentioned.

For the exoskeleton arm, singularities occur when joints 1 and 3 or joints 3 and 5 align with each other (see Figure 2). To minimise the frequency of this occurrence, the axis of joint 1 was positioned such that singularities with joint 3 take place only at locations that are anthropometrically hard to reach. To allow some user-specific flexibility in the design, the singular position is movable in $15^{\circ}$ increments. For the placement shown in Figures 1(c) and 1(d), the singularity can be reached through simultaneous extension and abduction of the upper arm by $47.5^{\circ}$ and $53.6^{\circ}$, respectively (see Figure 2(e)). Similarly, the same singularity can be reached through flexion and adduction by $132.5^{\circ}$ and $53.6^{\circ}$, respectively (see Figure 2(f)). The singularity between joints 3 and 5 naturally occurs only in full elbow extension, i.e. on the edge of the forearm workspace (Figure $2(\mathrm{~g})$ ). With each of these singularity vectors at or near the edge of the human workspace, the middle and majority of the workspace is free of singularities.

Another aspect to consider when placing singularities is mechanical isotropy. For optimal ease of movement in any direction, singular axes should be placed orthogonal to directions where isotropy is of highest importance. For the pointed singularity placement, isotropy will be maximised in $42.5^{\circ}$ of shoulder flexion and $26.4^{\circ}$ of shoulder 


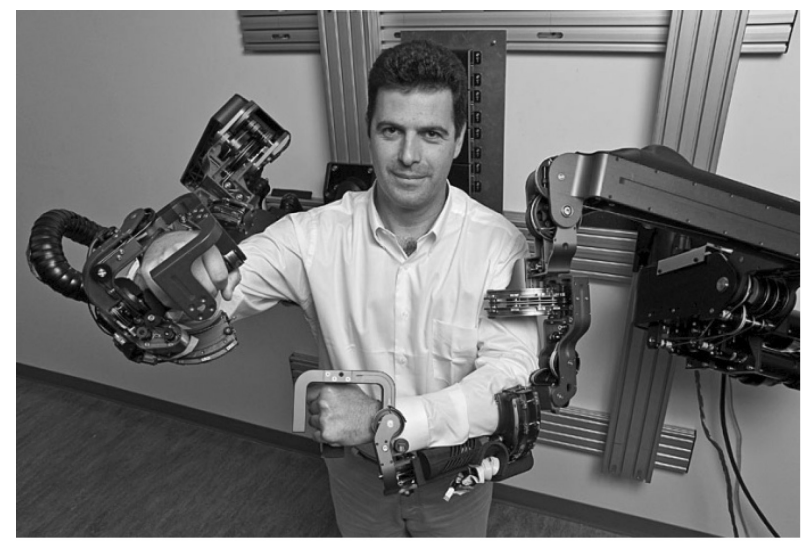

(a)

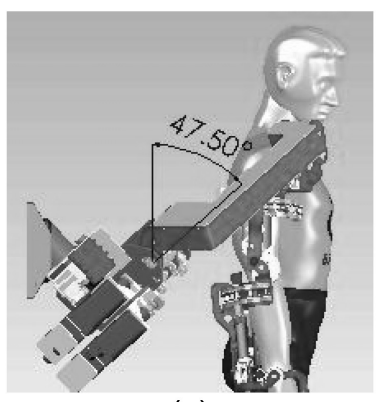

(c)

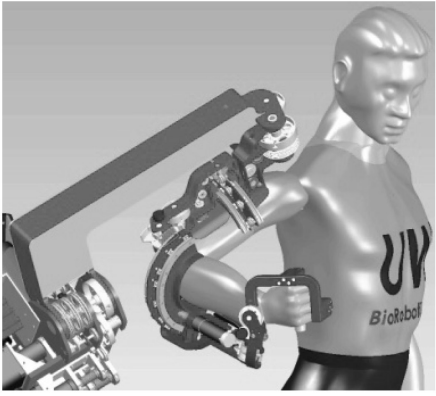

(e)

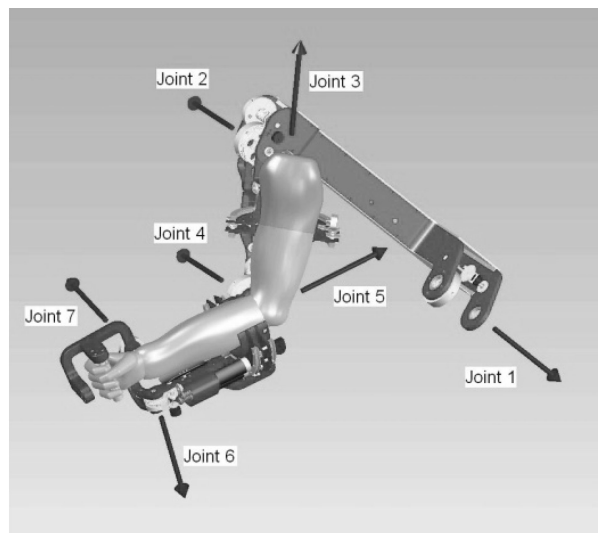

(b)

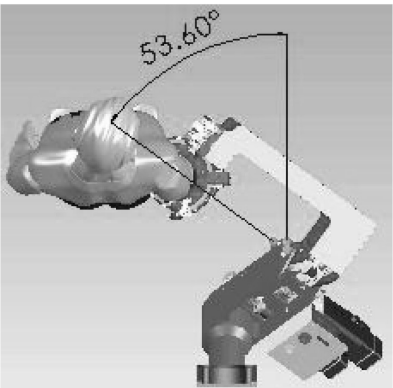

(d)

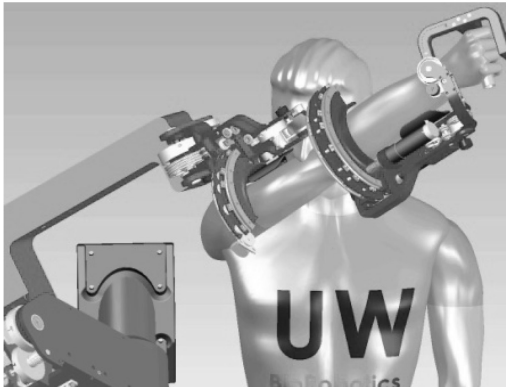

(f)

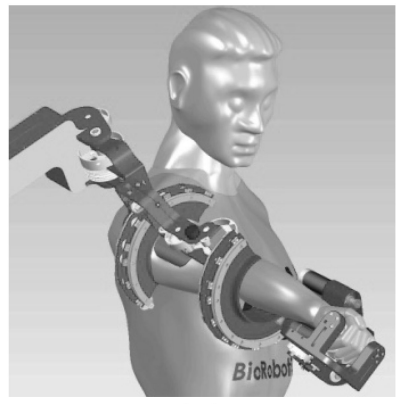

(g)

Figure 2. The Exo-UL7 a two-arm exoskeleton system with 7 DOFs in each arm. (a) System overview. (b) Rotation axes - definition. Orientation of the base shoulder frame (joints 1,2,3) to adjust the singular configuration of the shoulder joint. Mechanical Singularities - mechanical singularities between axes 1 and 3 occur around the shoulder internal-external rotation axis in configurations (e) and (f). A singularity between axes 3 and 5 also occurs in full elbow extension $(\mathrm{g})$.

abduction, values that lie in the median of the shoulder's range of motion (ROM) as assessed from the ADL study.

\section{Dynamics}

The human arm dynamics were studied using an analytical approach. A mathematical model of the human arm with 7 DOFs, consistent with the 7-DOF VICON model used during data collection, was developed using Mathematica (Wolfram Research, Inc.). The Power of Exponentials ap- proach (Murray and Sastry 1994) was used to eliminate potential singular effects at the shoulder, a common problem associated with the use of Euler angles. Analytical expressions of the seven equations of motion were derived from the model and converted to Matlab syntax for single-syntax post-processing.

The general form of the equation of motion is expressed as Equation (5).

$$
\tau=M(\Theta) \ddot{\Theta}+V(\Theta, \dot{\Theta}) \dot{\Theta}+G(\Theta),
$$


where $M(\Theta)$ is the $7 \times 7$ mass matrix, $V(\Theta, \dot{\Theta})$ is a $7 \times$ 1 vector of centrifugal and Coriolis terms, $G(\Theta)$ is a $7 \times$ 1 vector of gravity terms and $\tau$ is a $7 \times 1$ vector of the net toques applied at the joints. Given the kinematics of the human $\operatorname{arm}(\ddot{\Theta}, \dot{\Theta}, \Theta)$, the individual contributions on the net joint toque $(\tau)$ vector were calculated for every action of each subject, which allow one to examine the contribution of gravity in comparison to the effects on net torque from inertial, centrifugal and Coriolis forces.

Measured subject parameters (height, weight and segment lengths) were used to compute estimates for segment properties of mass and inertia according to Equations (68).

$$
\begin{aligned}
c m_{i} & =L_{i} \cdot c m_{i}(L), \\
m_{i} & =B W \cdot m_{i}(B W), \\
I_{i, j} & =m_{i} \cdot\left[K_{j}(L) \cdot L_{i}\right]^{2}
\end{aligned}
$$

where $L_{i}$ refers to the longitudinal length of the segment, $B W$ is the subject's bodyweight and $K(L)$ refers to the radius of gyration expressed as a linear function of the segment length. Similarly, on the right-hand side of Equations (6) and (7), the centre of mass is expressed as a linear function of the segment length, and mass as a linear function of bodyweight, with parameters taken from de Leva 1996. Subscripts $i$ and $j$ indicate the segment (upper arm, lower arm or hand) and coordinate axes of interest ( $\mathrm{x}, \mathrm{y}$ or $\mathrm{z}$ ), respectively. Equation (6) was used to calculate the longitudinal component of the centre of mass, while transverse and sagittal components were represented by average values from literature (Department of Transportation 1975). Mass and inertial properties of manipulated objects were small in comparison with the hand, and for this reason were neglected in the study. Inertial values of the hand were approximated by the values of an open hand taken from literature (Department of Transportation 1975; de Leva 1996; McConville et al. 1980) during all tasks.

\section{Results}

The results of the study reveal several phenomena expressed by the arm kinematics and dynamics while executing common activities of daily living, such as (1) the unique distribution of the human arm kinematics which is mapped with respect to isotropy, (2) the difference between position and orientation of the human arm during object manipulation, (3) the effect of the grasp type (normal and power) on the overall arm kinematics and (4) the distribution of the decomposed joint torque dynamics.

\section{A. ADL kinematic distribution of data}

Kinematic distribution of the entire database collected in this study is plotted in Figures 3 and 4 utilising polar coordinate systems. The plot in Figure 3 is a two-dimensional representation of a hemisphere. The centre of the hemisphere (the origin of the polar plot) is mapped to the centre of the humeral head. The polar graph in Figure 3 represents a projection of the hemisphere in the frontal plane. The plot is segmented into increments of $10^{\circ}$ by $10^{\circ}$ (azimuth and elevation). Figure 3(a) indicates a bimodal distribution with two distinct local maxima for the elbow of the right arm. One local maximum is when the arm is at $25^{\circ}$ to the right (azimuth - abduction) and $55^{\circ}$ down (elevation - flexion) and the other maximum is when the arm is at $35^{\circ}$ to the right (azimuth - abduction) and $35^{\circ}$ down (elevation - flexion). Figure 3(b) depicts the distribution of the spatial position of the wrist (azimuth and elevation) using the same coordinate system as shown in Figure 3(a). The distribution of the wrist joint location is bimodal with the highest frequency at $25^{\circ}$ to the left (azimuth) and $5^{\circ}$ down (elevation) - a position that translates to a location along the axis of symmetry of the human body almost at the height of the shoulder joint.

Figure 4(a) depicts the location of the elbow joint in a similar fashion to Figure 3(a). Contour lines of constant isotropy were added to this graph with increments of 0.1 . To display more clearly lines of isotropy alongside kinematic results in Figure 3(a), the isotropy values are subtracted from unity (1-isotropy). Therefore, in this figure an isotropy with a value of 1 represents a singular configuration of the exoskeleton arm that should be avoided. By reorienting the base of the exoskeleton, the singular configuration is relocated to the edge of the workspace. The spatial histogram of the elbow joint centre position (Figure 3(a)) indicates that during the day-to-day activities that were tested in the above protocol, the elbow never reaches the singular configuration of the exoskeleton arm. Moreover, majority of the shoulder manipulation in ADL tasks will result in exoskeleton isotropy values below 0.4. The most frequently used shoulder orientation is translated into an exoskeleton isotropy value which is equal to or greater than 0.8 (1-isotropy $\leq 0.2$ ).

The ADL data illustrated in Figure $4 \mathrm{~b}$ indicate a trimodal shape to the elbow angle distribution with modal centres at $40^{\circ}, 70^{\circ}$ and $130^{\circ}$ approximately. Normal distributions of the wrist joint angle in both flexion/extension as well as ulnar/radial deviation were observed and depicted in Figures 4(c) and 4(d), respectively, with the highest frequencies between $10^{\circ}$ and $25^{\circ}$ of wrist joint extension and $0^{\circ}$ and $5^{\circ}$ of wrist joint radial deviation. Superimposed in red over the ADL data is a measure of isotropy. In Figure 4(b), the line displays the corresponding isotropy of the combined shoulder/elbow complex across the range of elbow angles. For each distribution, the isotropy of a point in the centre of the handgrip is then calculated with respect to the shoulder, taking into account the three-dimensional movements of the proximal exoskeleton joints. For spatial clarity in Figures 4(b)-4(d), the value (1-isotropy) rather than isotropy is plotted, which means a value of ' 1 ' 

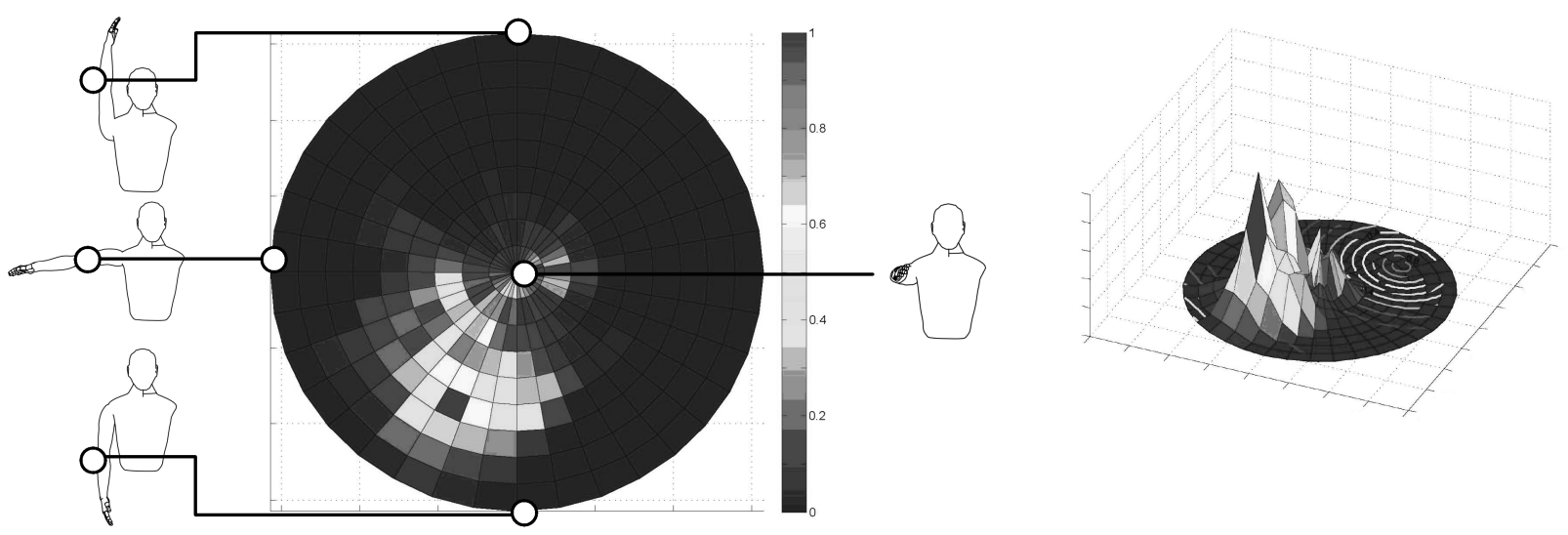

(a)
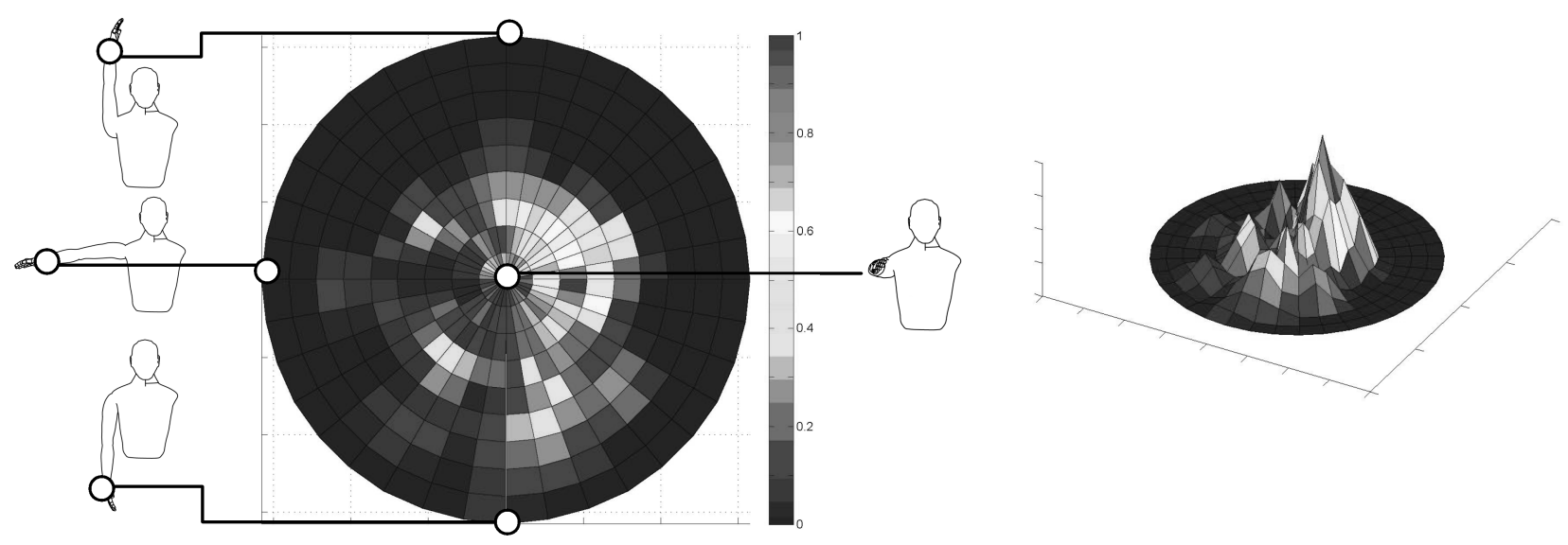

(b)

Figure 3. Histograms depicting the distribution of the elbow (a) and wrist (b) joint positions in a polar coordinate system with respect to the middle of the humerus. One may note that the distance between elbow joint and shoulder joint is fixed and dictated by the length of the upper arm; however the distance between shoulder joint and wrist joint varies and depends on elbow joint angle. This distance is omitted from (b). Key arm configurations and the corresponding locations on the graph are depicted. Colour represents the probability in which a specific joint location (elbow and wrist) was observed in the database.

represents the best isotropy and ' 0 ', the worst. In the case of elbow movement (Figure 4(b)), while the singularity occurs when the elbow is fully extended to $180^{\circ}$, the best isotropy occurs at an angle near $120^{\circ}$ of flexion (i.e. shoulder, elbow and end-effector form a near equilateral triangle) rather than at $90^{\circ}$ as would result from a two-dimensional analysis of the elbow. For wrist flexion/extension, optimal isotropy is around $0^{\circ}$, near the most commonly used region, whereas in wrist radial/ulnar deviation, the isotropy is unaffected by joint position.

\section{B. ADL dynamic distribution of data}

Dynamic distributions of the ADL data are illustrated in Figure 5. Distributions for overall torque are normal in shape with medians at $-1,-3.9,-1.4,-1.7,-0.01$, -0.14 and $-0.09 \mathrm{Nm}$, according to the previously defined joint convention. Figures 5(b) to 5(d) show the contribu- tions of various components to total torque during ADL tasks including the gravitational effects (Figure 5(b)), Coriolis and centrifugal effects (Figure 5(c)) and inertial effects (Figure 5(d)). From the figures, it is clear that gravitational loads are the primary components of total joint torques. In comparison to gravitational effects, the contributions to the total joint torque due to the arm acceleration and velocity are 1 and 2 orders of magnitude smaller in the shoulder (SA, SF, SR) and elbow (EF), respectively . At the wrist, the same ratios between components are approximately $1: 2$ and 1:100, while in pronosupination of the forearm, ratios are about 1:2 and 1:20, respectively. The data are tabulated in percentiles in Table 3 .

\section{Object manipulation: Positioning vs. orienting}

Kinematic results show task-specific differences in joint motion combinations. Four of the actions shown in 


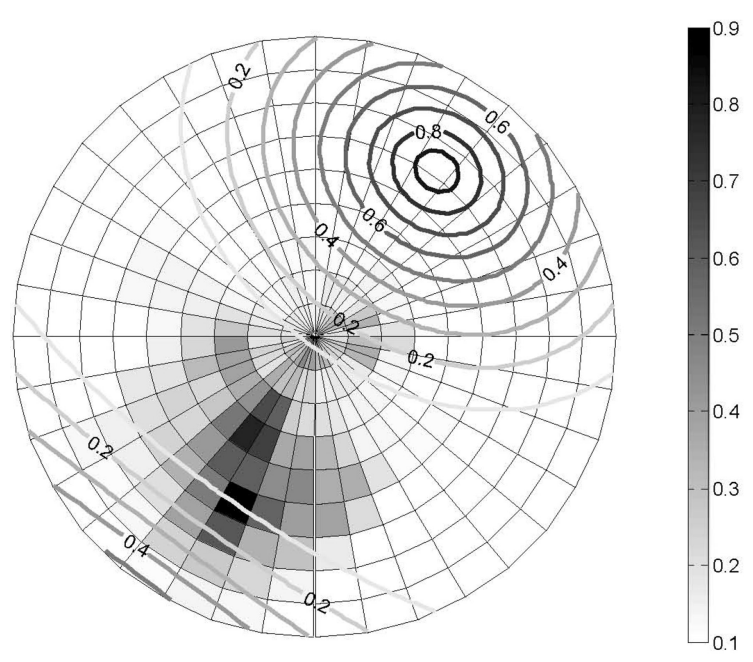

(a)

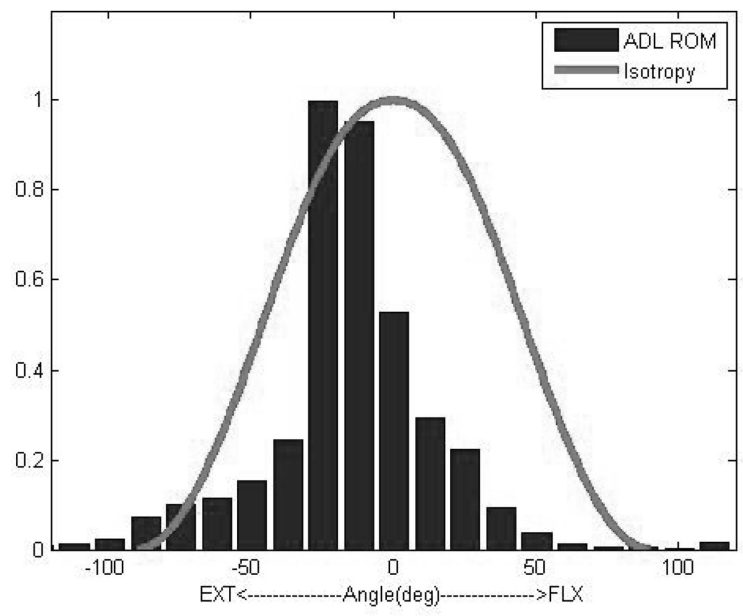

(c)

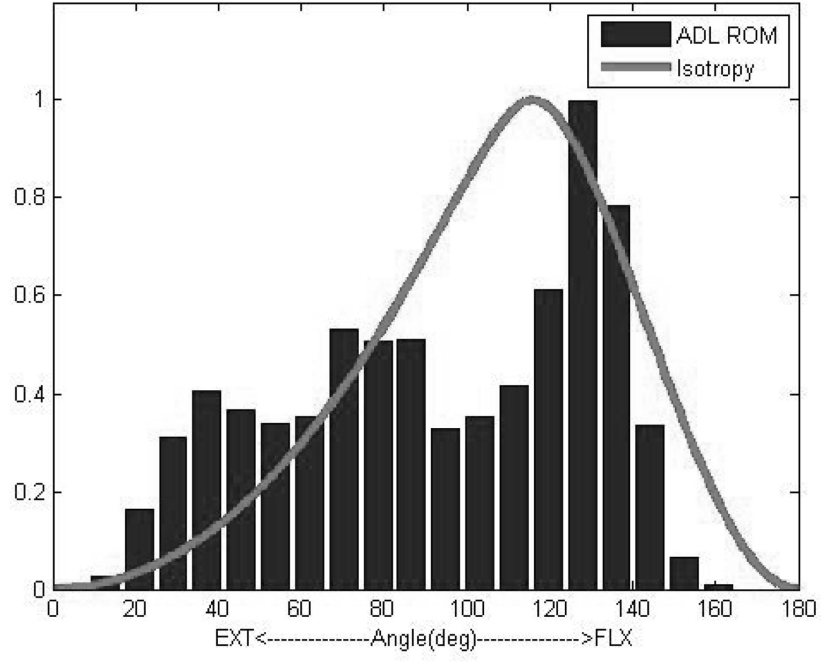

(b)

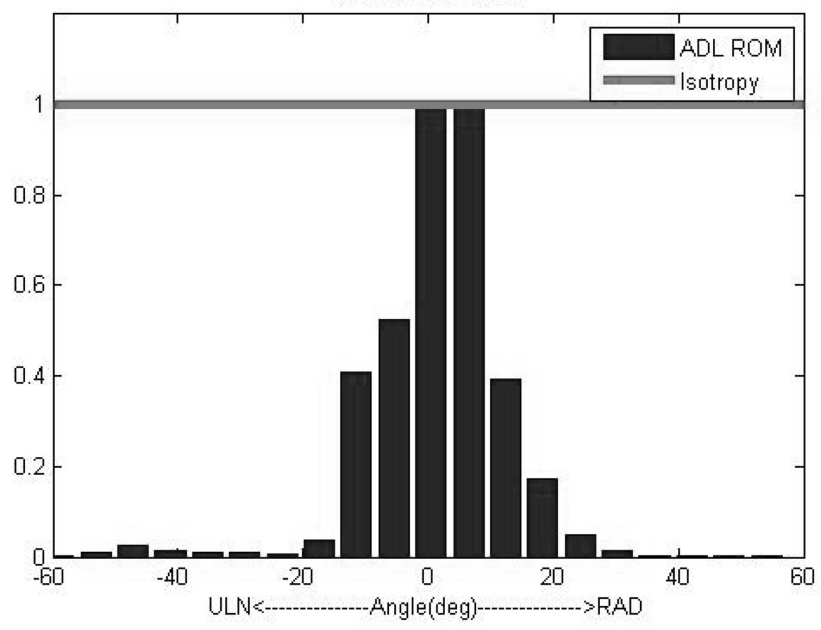

(d)

Figure 4. Histograms depicting the distribution of the elbow and wrist joints position and angles along with the exoskeleton arm isotropy. (a) Lines of constant isotropy are superimposed on the distribution of the elbow joint location in space with respect to the shoulder joint location (see figure $3 \mathrm{a}$ for details). The distribution of the joint angle along with the joint isotropy throughout the range of motion are shown for flexion/extension of the elbow (b), flexion/extension of the wrist (c) and radial/ulinar deviation. Note that for clarity in (a), the value of isotropy lines have been subtracted from unity (1-isotropy) such that the darker lines indicate regions of poorer performance.

Table 3. The 5th, 50th and 95th percentile values of position in degrees (top), and total torque in Nm (bottom), generated during daily activities at each of the 7 DOFs. Mean and SD are also provided for joint positions.

\begin{tabular}{|c|c|c|c|c|c|c|c|}
\hline & SA & SF & SR & $\mathrm{EF}$ & FR & WF & WD \\
\hline \multicolumn{8}{|l|}{ Position } \\
\hline Mean & -24.1 & -34.6 & 9.0 & -81.0 & 19.2 & -9.6 & 0.8 \\
\hline SD & 56.4 & 27.3 & 64.7 & 39.0 & 56.3 & 53.6 & 14.6 \\
\hline 5 th & -91.5 & -75.0 & -85.0 & -137.7 & -76.7 & -74.0 & -12.9 \\
\hline 50th & -24.0 & -36.0 & 8.7 & -83.7 & 22.2 & -13.7 & 1.0 \\
\hline 95th & 30.0 & 8.7 & 86.3 & -23.9 & 86.0 & 44.3 & 18.7 \\
\hline \multicolumn{8}{|l|}{ Total } \\
\hline 5 th & -4.65 & -10.89 & -3.80 & -4.07 & -0.85 & -0.46 & -0.39 \\
\hline 50th & -1.00 & -3.92 & -1.35 & -1.65 & -0.01 & -0.14 & -0.09 \\
\hline 95th & 3.33 & 1.71 & 0.53 & 0.92 & 0.72 & 0.21 & 0.25 \\
\hline
\end{tabular}



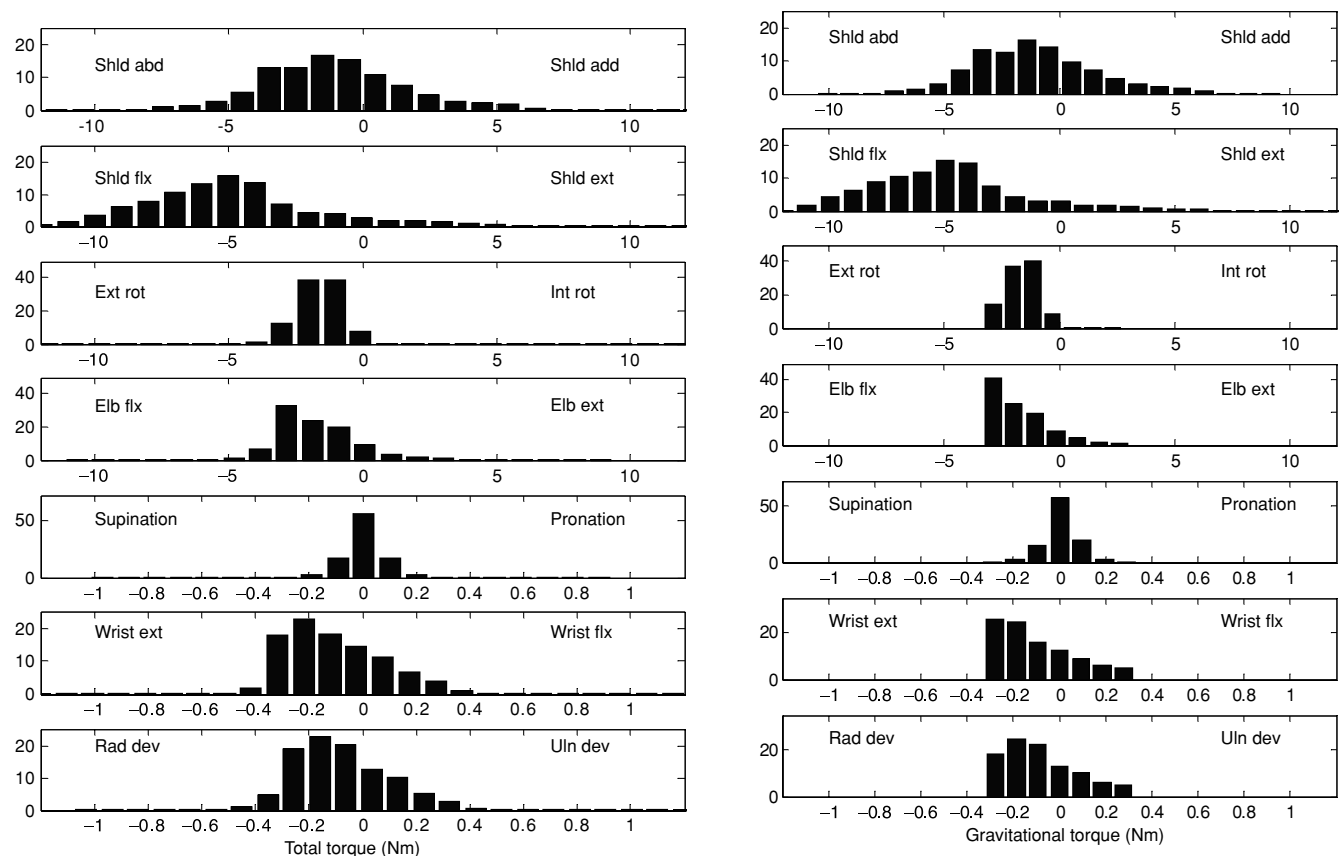

(a)

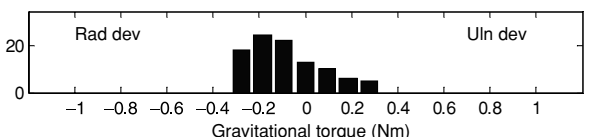

(b)
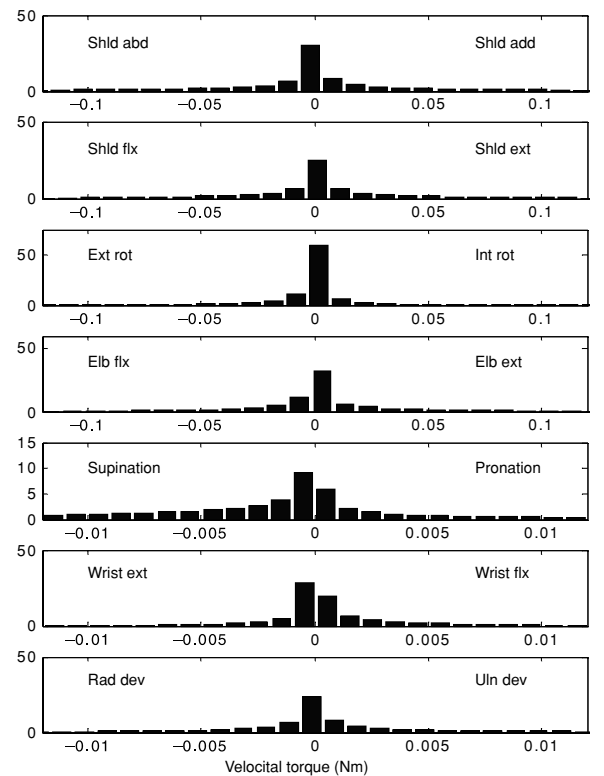

(c)

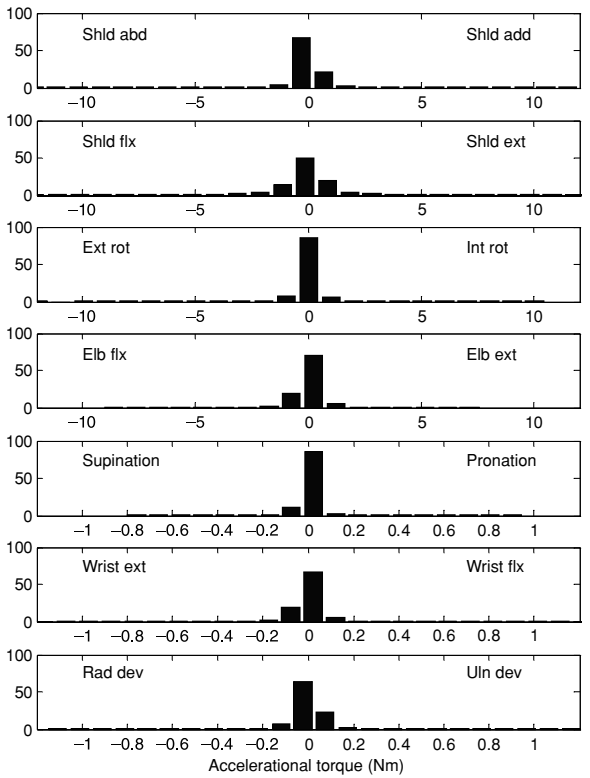

(d)

Figure 5. Histograms showing the distribution of joint torques, (a) Total joint torque $\tau$. (b) Joint torque due to gravitational loads $G(\Theta)$. (c) Torque due to velocity loads (Coriolis and centrifugal effects) $V(\Theta, \dot{\Theta})$. (d) Torque due to the inertial loads $M(\Theta) \ddot{\Theta}$. Each plot represents the complete set of data across all actions and subjects. All distribution graphs are plotted with x-axis of the joint torque and the distribution as percentage in the y-axis from top to bottom for SA, SF, SR, EF, FR, WF and WD.

Figure 6 show mean and SD curves from the following task categories: 2 reaching tasks (actions 2 and 3), 1 functional task (action 9) and 1 eating task (action 16). An immediate observation is the involvement of the hand during functional tasks as opposed to general reaching and positioning tasks. In actions 2 (Figure 6a) and 3 (Figure 6b), the task goal is to move an object from one location to another, in this case most of the joint movement as well as high velocity motion takes place in the shoulder and elbow in order to position the wrist, while there is minimal involvement of orientation of the wrist. In contrast to functional actions, such as opening a cupboard (Figure 6c) or eating 
Action 2: Moving an object between desk positions.
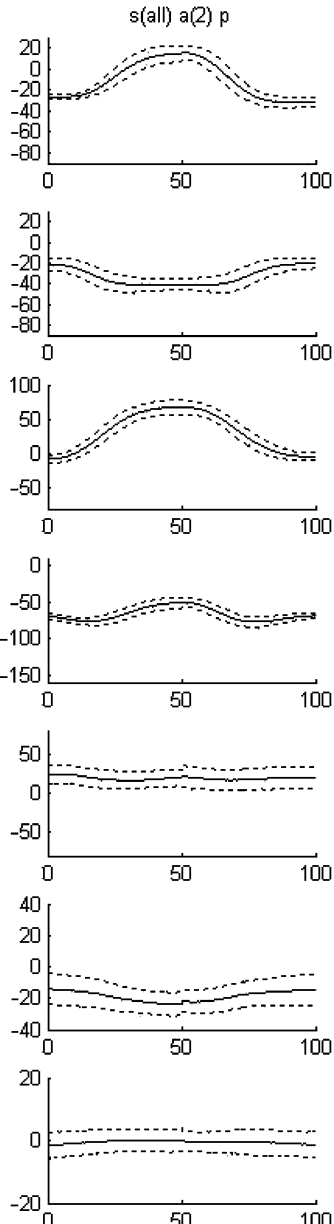

(a)
Action 3: Moving an object between shelf positions.
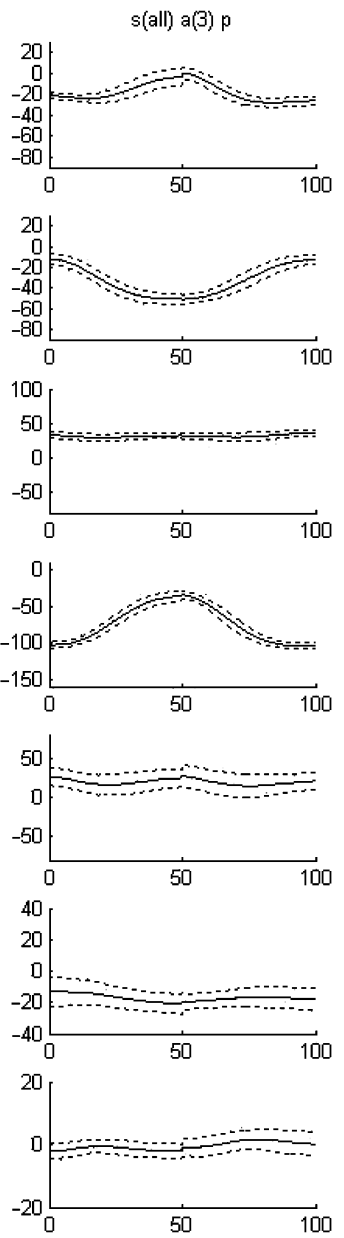

(b)
Action 9: Opening and Action 16: Eating with a

Action 16: Eating
fork (normal grasp) closing a cabinet.

$\mathrm{s}(\mathrm{all}) \mathrm{a(16)} p$
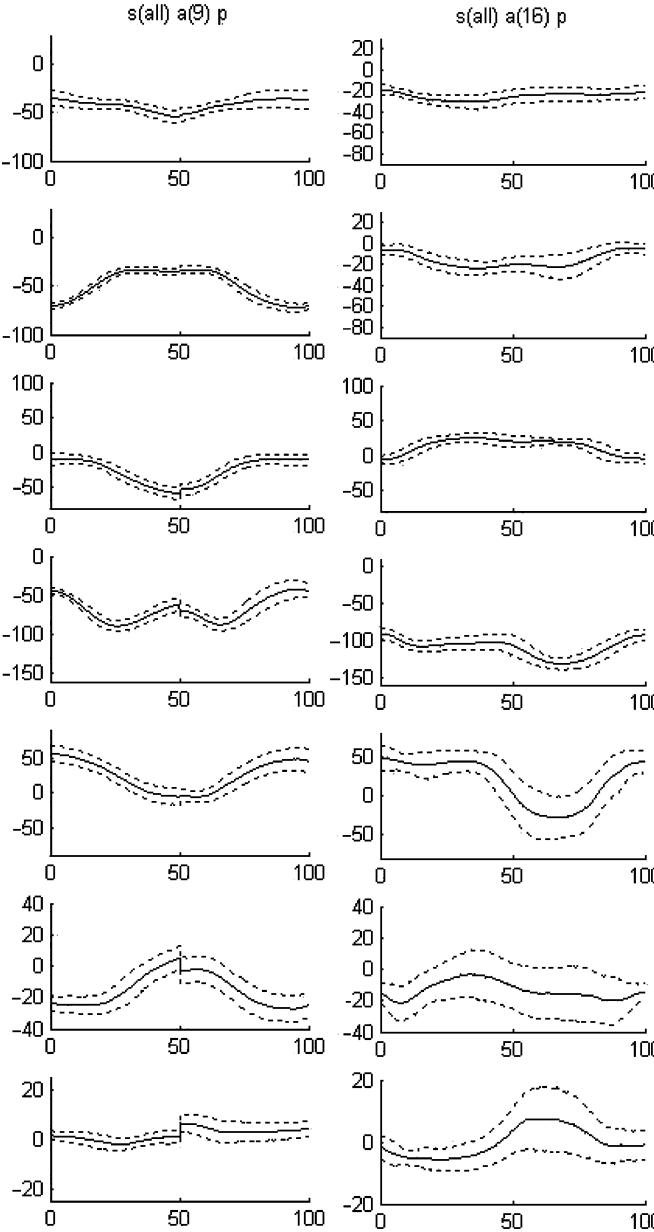

(c)
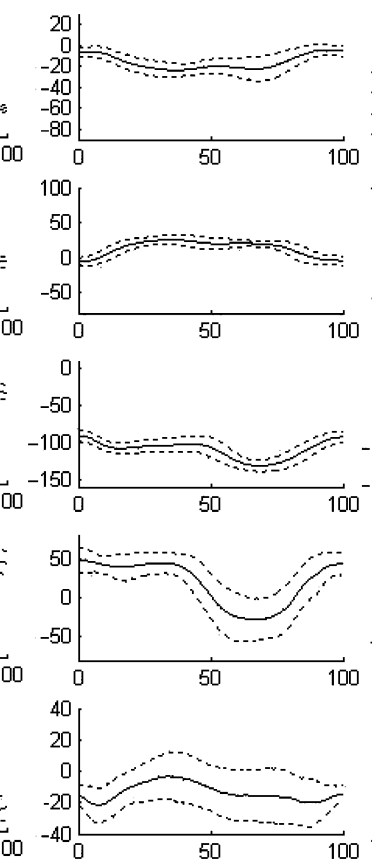

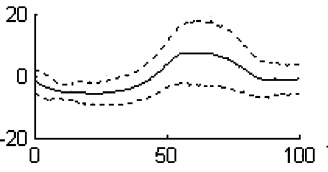

(d)
Action 17: Eating with a fork (power grasp)
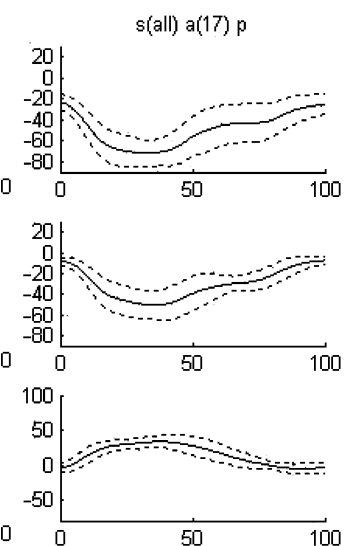
in
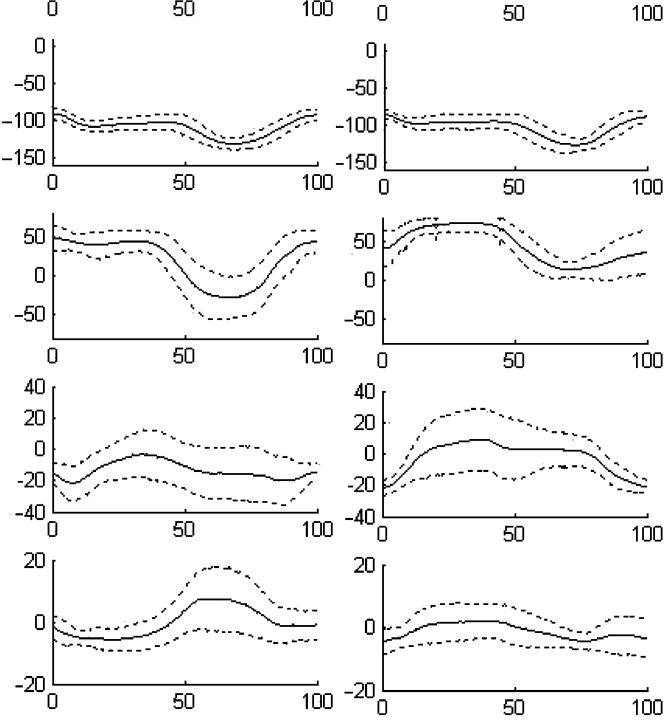

(e)

Figure 6. Mean (solid line), mean plus and mean minus SD (dash lines) for segmented position data averaged over six subjects, six trials each (36 trials total), shown for actions 2, 3, 9, 16 and 17, from left to right. All plot domains are shown from 0 to $100 \%$ of the segmented action, and ranges are given in degrees from top to bottom for SA, SF, SR, EF, FR, WF and WD.

with a fork (Figure 6d), during manipulation tasks much larger ROMs are seen in forearm rotation and both wrist flexion and deviation. Additionally, some complex tasks require moderate involvement across all seven arm joints (Figure 6c).

\section{Method of grasp: Normal vs. powered}

Figures 6(d) and 6(e) illustrate results from two types of fork handling: a typical three-fingered grasp (Figure 6(d)) and a powered grasp of a fork (Figure 6(e)) having a common handle buildup to aid in myopathic grasping. For impaired users with difficulty in achieving a firm grasp, the buildup lessens the amount of palmar flexion required to grasp the fork. Comparison of the plots at each joint reveal significant increases in shoulder abduction $(p<0.005)$ and shoulder flexion $(p<0.005)$ to accommodate food stab- bing as a result of the altered grasp. Additional trends showed increases in pronation, wrist flexion, and wrist radial deviation with the powered grasp. The results suggest that excessive shoulder movement is needed to compensate the loss of finger manipulation due to the power grasp.

\section{E. ADL task subgroup variations}

Separating ADL results by task subgroup shows significant similarities between both position (Figure 7) and torque (Table 4) distributions between subgroups. Differences between subgroup position distributions can be seen in shoulder and elbow data in terms of both modality of distributions and locations of modal centres. For example, a dominant bimodal elbow distribution appears in hygiene tasks, with modal centres that differ from those present in either reaching or functional tasks. The torque distribution 


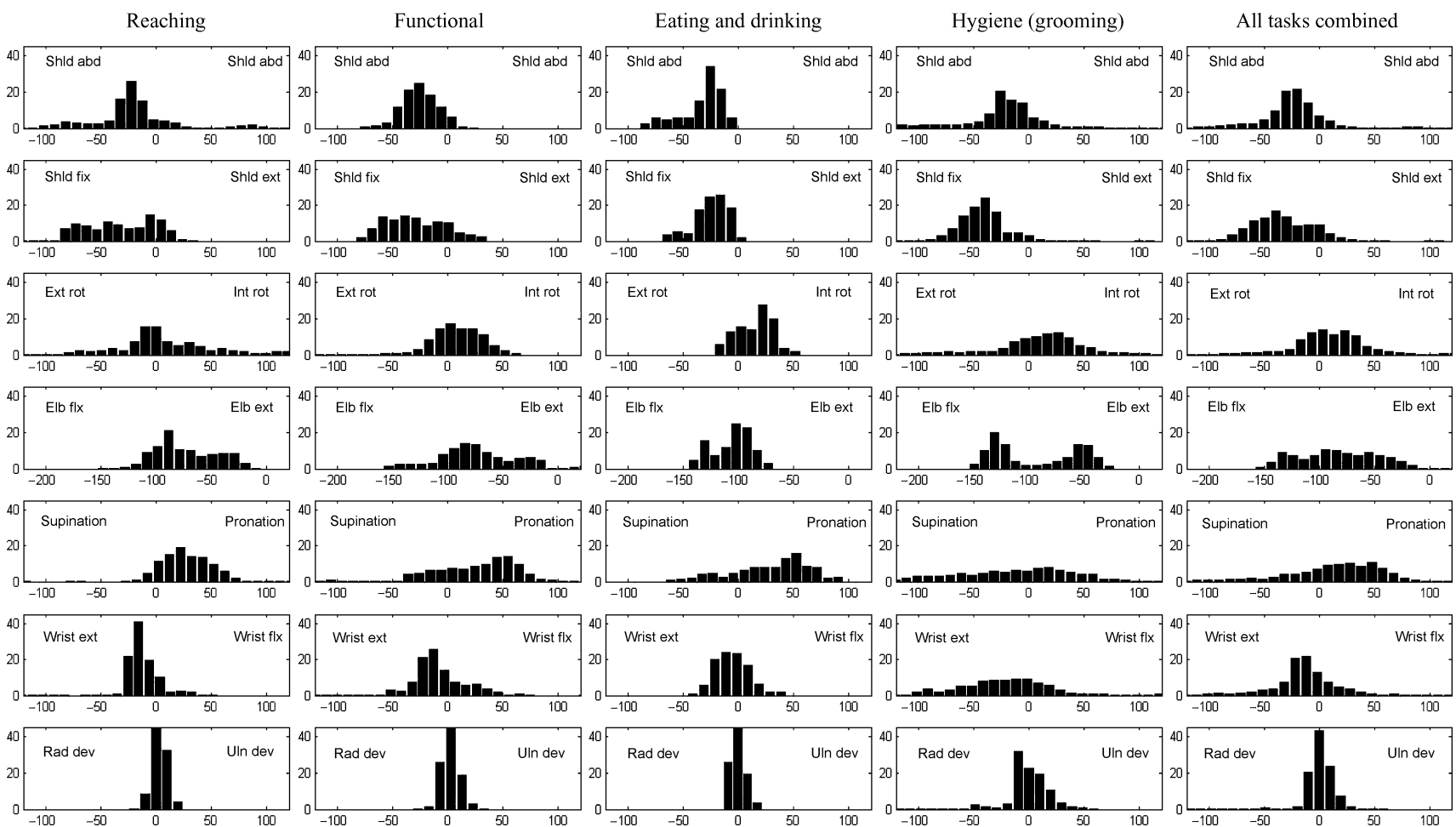

Figure 7. Joint angle histograms for ADLs are represented by activity subgroup for position data for each arm joint (from left to right: reaching, functional, eating and drinking, hygiene and all tasks combined). Distribution ranges are given as percentage time, from top to bottom, for SA, SF, SR, EF, FR, WF and WD. Horizontal axes are in degrees.

data (Table 4) show the more subtle differences in limb dynamics. To help elucidate the trends in torque ranges between action subgroups, a table of percentage differences was created in Table 5. The percentage difference was taken with respect to the values of the combined set of all tasks and then normalised by the range of torque (5th to 95th percentile) for the corresponding joint. In Table 5 , higher differences of $20-40 \%$ between individual

Table 4. 5th, 50th (median) and 95th percentile values of total joint torque (Nm) for each of the four task subgroups.

\begin{tabular}{|c|c|c|c|c|c|c|c|}
\hline & SA & SF & SR & $\mathrm{EF}$ & FR & WF & WD \\
\hline \multicolumn{8}{|c|}{ Reaching } \\
\hline 5 th & -4.65 & -10.78 & -3.49 & -4.28 & -0.58 & -0.47 & -0.39 \\
\hline 50th & -1.43 & -3.40 & -1.30 & -1.88 & -0.01 & -0.19 & -0.13 \\
\hline 95 th & 4.69 & 1.43 & 0.14 & -0.57 & 0.50 & -0.05 & 0.05 \\
\hline \multicolumn{8}{|c|}{ Functional } \\
\hline 5 th & -4.86 & -11.50 & -3.48 & -3.98 & -0.61 & -0.49 & -0.34 \\
\hline 50th & -1.13 & -4.15 & -1.24 & -1.64 & -0.01 & -0.17 & -0.06 \\
\hline 95th & 1.37 & 0.85 & 0.24 & 0.54 & 0.55 & 0.13 & 0.17 \\
\hline \multicolumn{8}{|l|}{ Eating } \\
\hline 5 th & -3.91 & -6.99 & -3.59 & -4.04 & -0.69 & -0.47 & -0.35 \\
\hline 50 th & -1.49 & -3.17 & -1.52 & -1.58 & 0.02 & -0.18 & -0.07 \\
\hline 95 th & 0.26 & -1.54 & -0.42 & -0.31 & 0.50 & 0.01 & 0.29 \\
\hline \multicolumn{8}{|l|}{ Hygiene } \\
\hline 5 th & -5.06 & -10.82 & -4.42 & -3.46 & -1.21 & -0.33 & -0.44 \\
\hline 50th & -0.48 & -4.55 & -1.49 & -1.32 & -0.05 & -0.01 & -0.08 \\
\hline 95 th & 3.99 & 3.64 & 1.60 & 1.76 & 1.08 & 0.34 & 0.40 \\
\hline \multicolumn{8}{|c|}{ All tasks } \\
\hline 5 th & -4.65 & -10.89 & -3.80 & -4.07 & -0.85 & -0.46 & -0.39 \\
\hline 50 th & -1.00 & -3.92 & -1.35 & -1.65 & -0.01 & -0.14 & -0.09 \\
\hline 95th & 3.33 & 1.71 & 0.53 & 0.92 & 0.72 & 0.21 & 0.25 \\
\hline
\end{tabular}


Table 5. Percentage differences between subgroup joint torques and combined (all tasks) joint torques at the 5th, 50th (median) and 95th percentile values. Percentage differences were calculated from Table 4 by subtracting the subgroup percentile values from the all task percentile values and dividing the result by the 95 th -5 th percetile torque range for the corresponding joint.

\begin{tabular}{|c|c|c|c|c|c|c|c|}
\hline & SA & SF & SR & $\mathrm{EF}$ & FR & WF & WD \\
\hline \multicolumn{8}{|c|}{ Reaching } \\
\hline 5 th & 0.02 & 0.86 & 7.26 & -4.26 & 17.49 & -1.93 & -0.33 \\
\hline 50th & -5.45 & 4.11 & 1.19 & -4.65 & 0.07 & -6.94 & -6.58 \\
\hline 95th & 16.99 & -2.21 & -8.86 & -29.88 & -14.24 & -39.56 & -31.90 \\
\hline \multicolumn{8}{|c|}{ Functional } \\
\hline 5 th & -2.65 & -4.89 & 7.27 & 1.71 & 15.45 & -4.40 & 7.33 \\
\hline 50 th & -1.58 & -1.84 & 2.62 & 0.12 & -0.17 & -3.78 & 3.80 \\
\hline 95th & -24.56 & -6.79 & -6.50 & -7.58 & -10.79 & -12.56 & -13.22 \\
\hline \multicolumn{8}{|l|}{ Eating } \\
\hline 5 th & 9.33 & 30.89 & 4.81 & 0.51 & 10.03 & -1.65 & 5.75 \\
\hline 50 th & -6.18 & 5.91 & -3.92 & 1.32 & 2.08 & -4.85 & 3.13 \\
\hline 95 th & -38.47 & -25.80 & -21.81 & -24.67 & -14.26 & -30.24 & 5.82 \\
\hline \multicolumn{8}{|l|}{ Hygiene } \\
\hline 5 th & -5.10 & 0.53 & -14.34 & 12.15 & -22.77 & 19.93 & -8.65 \\
\hline 50th & 6.50 & -5.02 & -3.18 & 6.49 & -2.22 & 19.21 & 1.36 \\
\hline 95th & 8.33 & 15.36 & 24.95 & 16.84 & 22.57 & 18.91 & 23.02 \\
\hline \multicolumn{8}{|c|}{ All tasks } \\
\hline 5 th & 0.00 & 0.00 & 0.00 & 0.00 & 0.00 & 0.00 & 0.00 \\
\hline 50th & 0.00 & 0.00 & 0.00 & 0.00 & 0.00 & 0.00 & 0.00 \\
\hline 95th & 0.00 & 0.00 & 0.00 & 0.00 & 0.00 & 0.00 & 0.00 \\
\hline
\end{tabular}

Percentage difference

$$
\begin{aligned}
& >30 \\
& >20 \\
& >10 \\
& 0-10
\end{aligned}
$$

subgroups and the complete dataset (all tasks) occur almost exclusively at the 95th percentile (rightmost bound) for most arm joints, while the 5th percentile values are generally within $10 \%$ of the torque range, with the exception of shoulder flexion while eating (Table 4). The 95th percentile torques are primarily composed of positive values of torque, which, for the defined frame orientations, act in the following directions of increasing motion: adduction, extension and internal rotation of the shoulder, elbow extension, forearm pronation, wrist flexion and wrist ulnar deviation. With most of torques at the 95th percentile being positive, the percentage differences at this percentile are almost all negative for reaching, functional and eating tasks. For hygiene tasks, however, the percentage differences at the 95th percentile with respect to the combined task set are positive, indicating that they have higher than average values for torques that generate motions in the directions defined as positive.

From Table 4, a second observation can be made. It is apparent that the values are primarily negative, meaning that the range of torques for each joint is shifted primarily to the left (negative range). Taking note of the directions of motion that were defined as negative, we notice that they are all in the direction that would oppose gravity in a typical reaching task, and thus require a negative torque from the subject in order to maintain a position of the arm in space. For the majority of daily activities, which require motions that are done at slow to moderate speed, this bias towards negative torques is prominent. However, one can notice that in the hygiene-related tasks, which include several higher-speed tasks (e.g. brushing the teeth, washing the face), this negative bias is less pronounced. One might argue that the higher-speed motions from the hygiene tasks require significantly higher positive torques than do the other task subgroups in order to accelerate the hand in the direction of gravity at a rate faster than $9.81 \mathrm{~m} / \mathrm{s}^{2}$. In reaching, eating/drinking and functional tasks, it seems that the subjects can rely on gravity alone to lower the arm, significantly reducing the positive torques that would otherwise be required. The tasks that perform faster motions in the direction of gravity, then in comparison accentuate these torques required by the subject.

\section{Discussion and conclusions}

The integration of human and robot into a single system offers remarkable opportunities for creating a new generation of assistive technology. Having obvious applications in rehabilitation medicine and virtual reality simulation, such 
a device would benefit members of both healthy and disabled populations. Despite the recent prominence of upper limb exoskeletons in assistive applications, the human arm kinematics and dynamics are usually described in single or multiple arm movements that are not directly associated with any concrete activity of daily living. Following this trend, the aims of the research reported in this manuscript have been (1) to fill a current gap in literature regarding the range and distribution of kinematics and dynamic of the human arm during daily activities and (2) to study the manipulability (isotropy) of a 7-DOF-powered exoskeleton arm given the kinematics and the dynamics of the human arm. As part of the study, both kinematics and dynamics of the dominant arm of six subjects were examined in a free and unconstrained environment. The 24 recorded daily activities, based on previous surveys, encompassed four archetypes of human arm manipulation, as general reaching, functional actions, eating and drinking and hygiene.

\section{A. Human arm kinematics during positioning and orienting tasks}

In various tasks, it has been observed that joint velocities and angular excursions tend to take place in one of several joint combinations. Combinations seem to be threefold, having either (a) high velocities in the shoulder and elbow, and low velocities in the wrist; (b) high velocities in the wrist, and low velocities in the shoulder and elbow or (c) high velocities in all three joints. Although some complex and partially constrained tasks, such as brushing the teeth or opening a cupboard, require the simultaneous use of shoulder, elbow and wrist joints, many tasks rely primarily on the use of the shoulder and elbow for positioning an object relative to the environment or positioning the hand relative to an object. But then, with the hand positioned in a desired location relative to the environment, the hand has been observed to perform high velocity manipulations in order to orient the hand and/or other objects with respect to one another. The implications on exoskeleton design are twofold. First, and for the majority of task training, the results support the separation of shoulder and elbow training from wrist training, yielding more simplistic robotic designs. Second, the results show that there are some tasks that require simultaneous joint control of both shoulder/elbow and wrist complexes. For these tasks, only exoskeleton devices having higher numbers of DOFs have the capacity to control and direct movement trajectories simultaneously across all three joints.

Additionally, it was noticed that even in healthy populations changing the rules of human-object interaction, such as the method of grasp for an eating utensil, resulted in compensatory strategies with more proximal joints. This suggests that the conditions of the subject populations may affect the joint ranges of motion needed for specific task training. Moreover, for compensatory strategies that may involve excessive sternoclavicular and scapular articulation, the stability of the human-machine interface with the arm is critical. Additional DOFs to support articulation about these joints can be added; however, keeping in mind the eventual goal of movement training (to produce normal joint trajectories), ultimately enforcing normal trajectories, requires sufficient stability in the human-machine interface, rather than through enabling the unnecessary, and sometimes undesired, motion.

\section{B. The exoskeleton isotropy and human arm kinematics during positioning and orienting tasks}

Since the exoskeleton is mechanically linked to the human body, its design must enable the human operator to utilise maximum of physiological workspace while avoiding singular configuration of the exoskeleton within this workspace. A singular configuration, also known as a 'gimbal lock', is common when a joint of a mechanism is structured with three rotational axes that intersect at a single point. Utilising this joint configuration is common in the design of exoskeleton systems which specifically target shoulder, wrist, hip and ankle joints, due to their physiological likeness to a ball and socket joint. Since the exoskeleton is usually mechanically linked to the human body, adjusting the singular configuration of its joints is essential during use in order to avoid singularities within the user's physiological workspace. By analysing the isotropy of the shoulder joint, as a performance index of the shoulder manipulability, and mapping it with respect to the spatial distribution of the human arm in ADL, the base of the exoskeleton was orientated so that the singular configuration of the exoskeleton shoulder joint is located as close as possible to the edge of the human arm's physiological workspace. As a result, the user's range of motion is not compromised and the region with the best isotropy is positioned to match the most common arm configuration during ADLs. Any exoskeleton shoulder joint design in which the rotation axes are placed along two of the three anatomical planes (sagittal, coronal or transverse) will lead to a singular configuration of the arm which in turn results in a gimbal lock position within the anatomical workspace of the shoulder - a situation that should be avoided.

\section{Gravitational dominance}

Comparing torque contributions from the effects of gravity $G(\Theta)$, centrifugal and Coriolis $V(\Theta, \dot{\Theta})$ and inertial loads $M(\Theta) \ddot{\Theta}$, it is clear that gravity is the dominant factor, having greater influence than the remaining factors combined. In particular, the effect of velocity-dependent terms of the equations of motion are usually two orders of magnitude below those dependent on position. Coincidentally, the velocity-dependent terms are computationally the most 
expensive terms in the equations of motion, contributing more than half of the total computation execution time. A favourable outcome of this result is that these computationally expensive terms can legitimately be removed from the equations of motion when it is used for real-time compensation. As a result, a significant reduction in the execution times of command signals would be achieved without a significant negative impact on the system performance. Moreover, the dominance of the gravitational loads may provide insight on the type of therapy provided to the disabled population. These results support the use and development of new strategies for compensation and gradual reintroduction of gravity. While the results also suggest that passive gravity balancing may offer substantial benefit in training ADL tasks, it is also acknowledged that 7-DOF gravity balancing is highly complex and non-linear. Therefore exoskeleton systems, particularly those designed for specific tasks (small ranges of motion), may benefit from passive gravity balancing mechanisms where the operating range will be centred in the region of highest linearity. For exoskeleton systems that require a large (human-like) workspace, development of passive gravity compensation mechanisms presents a much higher challenge. For the current prototype, we have elected to design for a purely active configuration with the potential for passive additions in the future.

\section{Subgroup torques}

As was noted in the results, reaching, functional and eating/drinking tasks yielded significantly lower positive torques than hygiene tasks, on average 1-3 Nm lower for shoulder and elbow joints and 0.3-0.5 Nm lower for wrist joints. Recall that the torque directions defined as positive in the study are shoulder adduction, shoulder extension, shoulder internal rotation, elbow extension, forearm pronation, wrist flexion and wrist ulnar deviation. For the majority of daily activities, where the arm is placed in front of the body to move or manipulate an object, it would generate torques of the opposite sign to counteract the forces of gravity. Clearly then, the elevated levels of positive torque in hygiene tasks are due to elevated accelerations of limb segments in the direction of gravity.

Exoskeletons offer promising capabilities for the rehabilitation and training of a number of motor deficits stemming from cardiovascular (stroke), orthopaedic (trauma) and neuromuscular pathologies. It is believed that the data presented in this manuscript can help in the design and development of new technologies to address this growing need.

\section{Acknowledgement}

This research is supported by in part by NSF Grant \# 0208468 entitled "Neural control of an upper limb exoskeleton system" and by TATRC Grant \# W81XWH-07-1-0715 entitled "The myoprocessor - muscle modeling for neural control of upper limb powered prosthetics and orthotics".

\section{References}

Banala SK, Kulpe A, Agrawal SK. 2007. A powered leg orthosis for gait rehabilitation of motor-impaired patients. IEEE International Conference of Robotics and Automation, April 2007. PDF; Rome, Italy.

Bergamasco M, Frisoli A, Avizzano CA. 2007. Exoskeletons as man-machine interface systems for teleoperation and interaction. In: Advanced in telerobotics. Berlin/Heidelberg: Springer.

Blaya A, Herr H. 2004. Adaptive control of a variable Impedance ankle-foot orthosis to assist drop-foot gait. IEEE Trans Neural Syst Rehabil Eng. 12(1):24-31.

Carignan CR, Naylor MP, Roderick SN. 2008. Controlling shoulder impedance in a rehabilitation arm exoskeleton. IEEE International Conference on Robotics and Automation Pasadena; May 19-23, 2008; California, USA.

Casolo F, Cinquemani S, Cocetta M. 2008. On active lower limb exoskeletons actuators. Proceedings of the 5th International Symposium on Mechatronics and its Applications (ISMA08); 2008 May 27-29; Amman, Jordan.

de Leva, P. 1996. Adjustments to Zatsiorsky-Seluyanov's segment inertia parameters. J Biomech. 29(5):12223-12230.

Department of Defense. 1991. Military handbook anthropometry of US military personnel, DOD-HDBK-747A, 1991.

Department of Transportation. 1975. Investigation of Internal Properties of the Human Body, DOT HS-801 430, 1975.

Ettore C, Rosen J, Perry JC, Burns S. November 2006. Myoprocessor for neural controlled powered exoskeleton arm. IEEE T Bio-Eng. 53(11):2387-2396.

Ferris DP, Sawicki GS, Daley MA. 2007. A physiologist's perspective on robotic exoskeletons for human locomotion. Int $\mathbf{J}$ Humanoid Rob. 4:507-528.

Guizzo E, Goldstein H. 2005. The rise of body boots. IEEE Spectrum. Vol 42, number 10, Oct 2005.

Jacob R, Brand M, Fuchs MB, Arcan M. 2001. A myosignalbased powered exoskeleton system. IEEE Trans Syst Man Cybern-Part A: Syst Hum. 31(3):10-222.

Jacob R, Fuchs MB, Arcan M. 1999. Performances of hill-type and neural network muscle models-towards a myosignalbased exoskeleton. Comp Biomed Res. 32(5):415439.

Jacob R, Perry JC. 2007. Upper limb-powered exoskeleton. J Humanoid Rob. 4(3):1-20.

Kawamoto H, Kanbe S, Sankai Y. 2003. Power assist method for HAL-3 estimating operator's intention based on motion information. Proceedings of the 2003 IEEE International Workshop on Robot and Human Interactive Communication; Millbrae, CA, USA.

Kiguchi K, Rahman M, Sasaki M. 2006. Neuro-fuzzy-based motion control of a robotic exoskeleton: considering endeffector force vectors. ICRA 2006; Orlando, FL, USA. 31463151.

Klein J, et al. 2008. Biomimetic orthosis for the neurorehabilitation of the elbow and shoulder (BONES). IEEE Biorob2008; Scottsdale, AZ, USA. 535-541.

McConville JT, Clauser CE, Cuzzi J. 1980. Anthropometric relationships of body and body segment moments of inertia. Dayton, OH: Aerospace Medical Research Laboratory, WrightPatterson Air Force Base. Report No. AFAMRL-TR-80-119. 
Mihelj M, Nef T, Riener R. 2007. ARMin II-7 DoF rehabilitation robot: mechanics and kinematics. ICRA 2007; Rome, Italy. 4120-4125.

Murray RM, Sastry SS. 1994. A mathematical introduction to robotic manipulation. Boca Raton, FL: CRC Press.

Perry JC, Rosen J. 2006a. Design of a 7 degree-of-freedom upperlimb powered exoskeleton. Proceedings of 1st International Conference on Biomedical Robotics and Biomechatronics; 2006 Feb 20-22; Pisa, Italy.

Perry JC, Rosen J. 2006b. Design of a 7 degree-of-freedom upperlimb powered exoskeleton. Proceedings of the 2006 BioRob Conference; 2006 Feb; Pisa, Italy.

Perry JC, Rosen J, Burns S. 2007. Upper-limb powered exoskeleton design. IEEE Trans Mechatron. 12(4):408-417.

Pons JL. 2007. Wearable robots: biomechatronic exoskeletons. San Francisco, CA: Wiley.

Ramanathan R, Stroud S, Alexander MD. 1994. Powered orthosis project forum. ASEL Tech Rep. TR \# ROB9405. (Online)

Romilly DP, Anglin C, Gosine RG, Hershler C, Raschke SU. 1994. A functional task analysis and motion simulation for the development of a powered upper-limb orthosis. IEEE Trans Rehabil Eng. 2(3):119-129.

Rosen J, Perry JC, Manning N, Burns S, Hannaford B. 2005. The human arm kinematics and dynamics during daily activities - toward a 7 DOF upper limb powered exoskeleton.
Proceedings of 12th International Conference on Advanced Robotics, 2005 July 18-20. Seattle, WA, USA, p. 532-539.

IJHR. 2007. Wearable robotics. Int JHumanoid Rob (IJHR). 4(3) (Sep, special issue)

Stanger CA, Anglin C, Harwin WS, Romilly DP. 1994. Devices for assisting manipulation: a summary of user task priorities. IEEE Trans Rehabil Eng. 2(4):256-265.

Stienen AHA, Hekman EEG, Van der Helm FCT, Prange GB, Jannink MJA, Aalsma AMM, Van der Kooij H. 2007. Dampace: dynamic force-coordination trainer for the upper extremities. IEEE 10th International Conference on Rehabilitation Robotics (ICORR) 2007; Kyoto, Japan.

Thom T, et al. 2006. Heart disease and stroke statistics-2006 update. AHA statistical update; Circulation. J Amer Heart Assoc. 113(6):e85-e151.

Tsagarakis NG, Caldwell DG. 2003. Development and control of a "Soft-Actuated" exoskeleton for use in physiotherapy and training. Auton Robots. 15(1):21-33.

US Census Bureau. 2001. Americans with disabilities: 1997; p. 3.

Van der Loos HFM, Reinkensmeyer DJ. 2008. Rehabilitation and health care robotics, handbook of robotics. New York, NY: Springer; p. 1223-1251.

Zoss A, Kazerooni H, Chu A. 2005. On the mechanical design of the Berkeley lower extremity exoskeleton (BLEEX). Proceedings of IEEE International Conference on Robotics and Automation, Barcelona, Spain. April 18-22, 2005. 

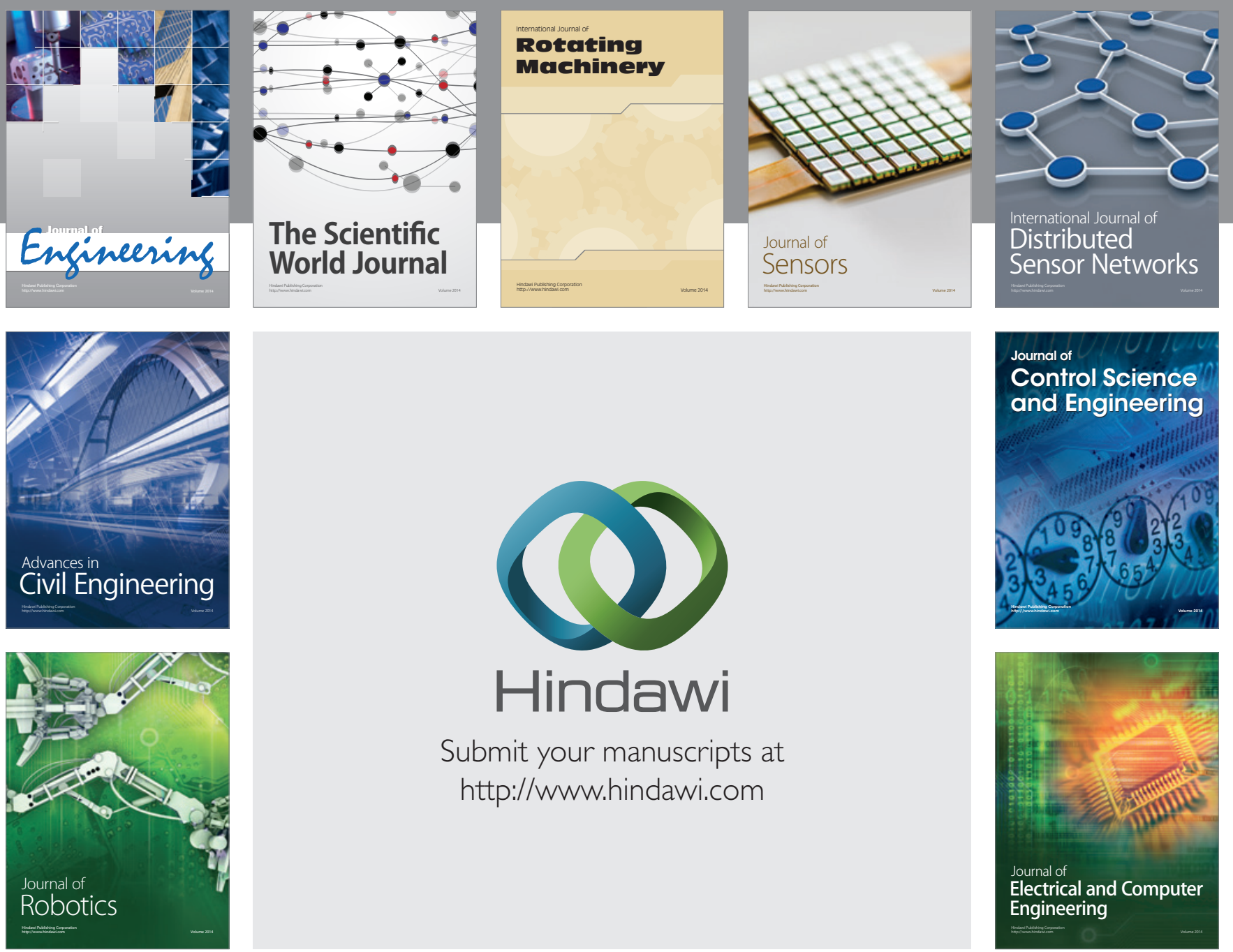

Submit your manuscripts at

http://www.hindawi.com
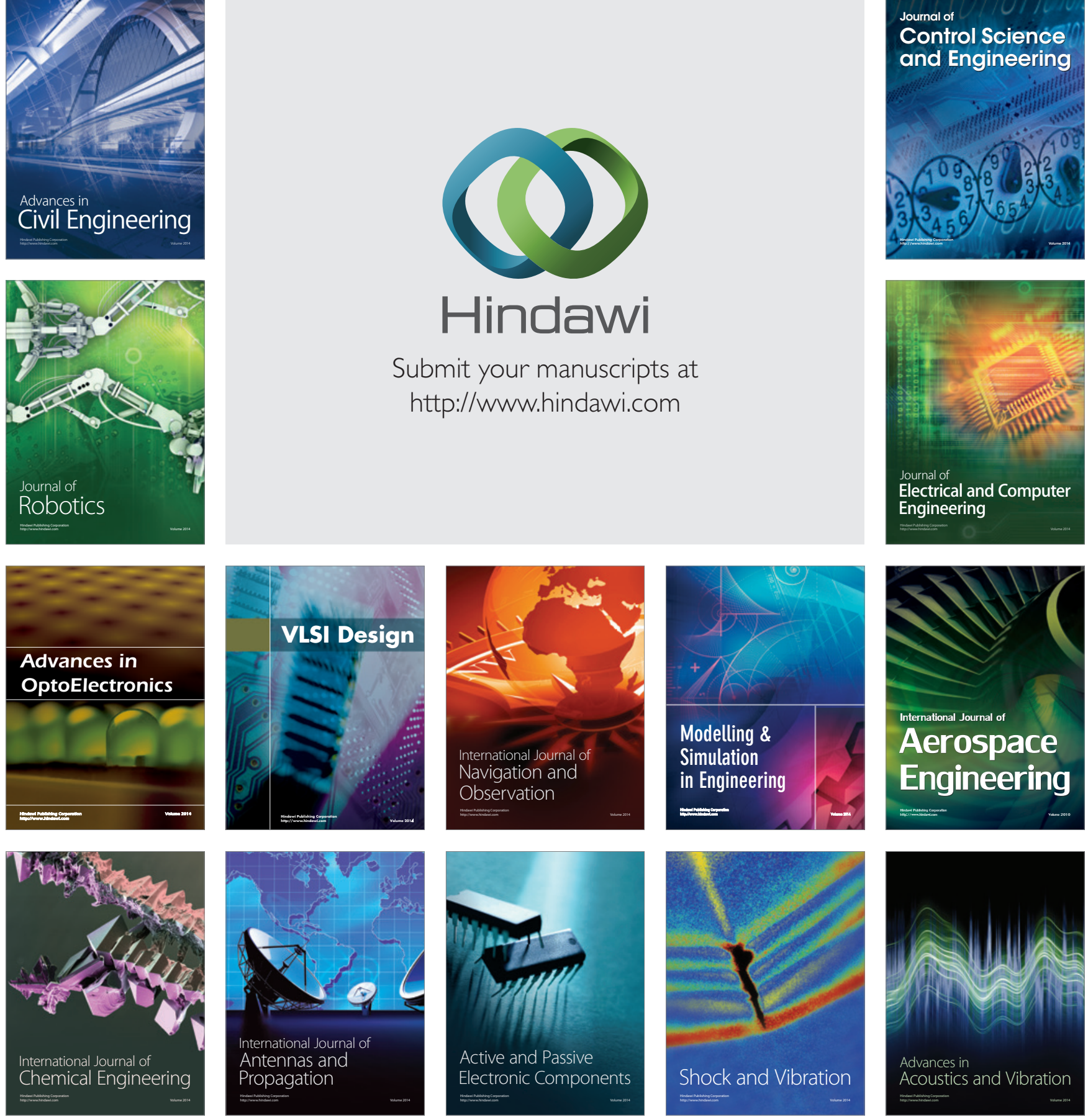